This item was submitted to Loughborough's Research Repository by the author.

Items in Figshare are protected by copyright, with all rights reserved, unless otherwise indicated.

\title{
Critical control in transcritical shallow-water flow over two obstacles
}

PLEASE CITE THE PUBLISHED VERSION

http://dx.doi.org/10.1017/jfm.2015.485

\section{PUBLISHER}

(c) Cambridge University Press

\section{VERSION}

AM (Accepted Manuscript)

\section{PUBLISHER STATEMENT}

This work is made available according to the conditions of the Creative Commons Attribution-NonCommercialNoDerivatives 4.0 International (CC BY-NC-ND 4.0) licence. Full details of this licence are available at: https://creativecommons.org/licenses/by-nc-nd/4.0/

\section{LICENCE}

CC BY-NC-ND 4.0

\section{REPOSITORY RECORD}

Grimshaw, Roger H.J., and Montri Maleewong. 2019. "Critical Control in Transcritical Shallow-water Flow over Two Obstacles". figshare. https://hdl.handle.net/2134/19523. 


\title{
Critical control in transcritical shallow-water flow over two obstacles
}

\author{
Roger H.J. Grimshaw ${ }^{1}$ and Montri Maleewong ${ }^{2}$ \\ ${ }^{1}$ Department of Mathematical Sciences, Loughborough University, \\ Loughborough LE11 3TU, UK \\ 2 Department of Mathematics, Faculty of Science, Kasetsart University, \\ Bangkok, 10900, Thailand
}

\begin{abstract}
The nonlinear shallow-water equations are often used to model flow over topography In this paper we use these equations both analytically and numerically to study flow over two widely separated localised obstacles, and compare the outcome with the corresponding flow over a single localised obstacle. Initially we assume uniform flow with constant water depth, which is then perturbed by the obstacles. The upstream flow can be characterised as subcritical, supercritical, and transcritical respectively. We review the well-known theory for flow over a single localised obstacle, where in the transcritical regime the flow is characterised by a local hydraulic flow over the obstacle, contained between an elevation shock propagating upstream and a depression shock propagating downstream. Classical shock closure conditions are used to determine these shocks. Then we show that the same approach can be used to describe the flow over two widely spaced localised obstacles. The flow development can be characterized by two stages. The first stage is the generation of upstream elevation shock and downstream depression shock from each obstacle alone, isolated from the other obstacle. The second stage is the interaction of two shocks between the two obstacles, followed by an adjustment to a hydraulic flow over both obstacles, with criticality being controlled by the higher of the two obstacles, and by the second obstacle when they have equal heights. This hydraulic flow is terminated by an elevation shock propagating upstream of the first obstacle and a depression shock propagating downstream of the second obstacle. A weakly nonlinear model for sufficiently small obstacles is developed to describe this second stage. The theoretical results are compared with fully nonlinear simulations obtained using a well-balanced finite volume method. The analytical results agree quite well with the nonlinear simulations for sufficiently small obstacles.
\end{abstract}

\section{Introduction}

\subsection{Background}

Shallow water flow of a homogeneous fluid over bottom topography is a fundamental problem in fluid mechanics and has been heavily studied from various points of view. A widely used approach when the topography is a single localised obstacle is the application of hydraulic concepts which lead to the classification of the flow in terms of the value of the upstream Froude number, defined as the ratio of the uniform upstream flow to the linear long wave speed. The flow is then described as supercritical, subrcritical or transcritical depending on whether the upstream Froude number is greater than unity, less than unity, or close to unity respectively, see for instance the monograph by Baines (1995) for a comprehensive account of hydraulic theory and the issues involved. In the supercritical case waves generated by the flow interaction with the obstacle propagate downstream away from the obstacle, and the flow at the obstacle location is a locally steady elevation. In the subcritical case, waves 
propagate upstream and downstream away from the obstacle, and the flow at the obstacle location is a locally steady depression. When wave dispersion is considered, steady lee waves are also formed downstream of the obstacle. Both these cases can be well understood, at least qualitatively, using linearised theory.

However, linearized theory fails in the transcritical regime, which is the main interest here, and then a nonlinear theory is needed to describe the locally steady hydraulic flow over the obstacle, which has an upstream elevation and a downstream depression, each terminated by upstream and downstream propagating undular bores. A popular model here in the weakly nonlinear regime when the obstacle has a small amplitude is the forced Korteweg-de Vries (KdV) equation, see Akylas (1984), Cole (1985), Grimshaw and Smyth (1986), Lee et al. (1989), Binder et al. (2006), Grimshaw et al. (2007) and the recent review by Grimshaw (2010). Various aspects of the extension to finite amplitudes in the long wave regime can be found in El et al. (2006), El et al. (2008), and El et al. (2009).

Thus transcritical shallow water flow is quite well understood for a single localised obstacle, but there have been comparatively very few studies of the analogous case when the there are two widely separated localised obstacles. In the context of this article the most relevant is the article by Pratt (1984) where a combination of steady hydraulic theory, numerical simulations using the nonlinear shallow water equations and laboratory experiments are used to infer that the formation of dispersive waves between the obstacles is needed to obtain a stable solution. More recently Dias and Vanden-Broeck (2004) Ee et al. (2010, 2011) have examined the possible presence of such waves for steady flows, while Grimshaw et al. (2009) considered the related problem of unsteady flow over a wide hole. Thus a new feature of interest when considering two obstacles is that the waves generated by each obstacle may interact in the region between the two obstacles, and then the question is how this interaction might affect the long-time outcome. In this paper we examine this scenario using the nonlinear shallow water equations, so that although finite-amplitude effects are included, wave dispersion is neglected and the generated waves are represented as shock waves. Our emphasis is on the transcritical regime for two widely-spaced localised obstacles. The nonlinear shallow water equations are solved numerically using a well-balanced finite volume method, and the results are shown in section 3. The simulations are supplemented by a combination of fully nonlinear hydraulic theory with classical shock closure conditions, and a reduced model used in the weakly nonlinear regime, presented in section 2 . We conclude in section 4.

\subsection{Formulation}

The basic model is one-dimensional shallow-water flow past topography, in which the flow is described by the total local depth $H$ and the depth-averaged horizontal velocity $U$. The upstream flow is a constant horizontal velocity $V>0$, and the forcing is due to a localised topographic obstacle $f(x)$ so that the bottom is at $z=-h+f(x)$ where $h$ is the undisturbed depth at infinity. Henceforth, we use non-dimensional coordinates, based on a length scale

$h$, a velocity scale $\sqrt{g h}$ and a time-scale of $\sqrt{h / g}$., in terms of which the equation system is

$$
\begin{gathered}
\zeta_{t}+(H U)_{x}=0, \quad H=1+\zeta-f, \\
U_{t}+U U_{x}+\zeta_{x}=0 .
\end{gathered}
$$

In these non-dimensional coordinates, the constant upstream flow is $F=V / \sqrt{g h}$, the Froude number. Here the topography $f(x)$ consists of two obstacles, each symmetrical, and placed 
a distance $L$ apart, with respective maximum heights (or depths) of $\epsilon_{1,2}$. Our interest here is when $\epsilon_{1,2}>0$, and the situation when either or both $\epsilon_{1,2}<0$ will be considered elsewhere. We assume that the separation distance $L$ is much greater than the width of the obstacles. Then the main parameters are the Froude number $F$, and the maximum heights $\epsilon_{1,2}$. This system is to be solved with the initial conditions

$$
H=1, U=F, \quad \text { at } t=0 .
$$

This is equivalent to introducing the obstacles instantaneously at $t=0$ into a constant flow. The solution will initially develop smoothly, but being a nonlinear hyperbolic system, we can expect the development of discontinuities in the derivatives of $\zeta, U$. The classical procedure is then to introduce shocks, given by

$$
-S[\zeta]+[H U]=0, \quad-S[H U]+\left[H U^{2}+\frac{1}{2} H^{2}\right]=0 .
$$

Here $S$ is the shock speed, and $[\cdots]$ denotes the jump across the shock. In the absence of the bumps $(f(x)=0)$, these classical shocks conserve mass and momentum.

In the transcritical regime when $F \approx 1$, it will be useful also to consider a weakly nonlinear model for small-amplitude topography, given by

$$
-\zeta_{t}-\Delta \zeta_{x}+\frac{3}{2} \zeta \zeta_{x}+\frac{f_{x}}{2}=0, \quad \Delta=F-1
$$

Here $U=F+u$ and $u \approx-\zeta$. The reduced model (5) can be seen as a dispersionless forced $\mathrm{KdV}$ equation, see the afore-mentioned references. For convenience we present an alternative derivation in the Appendix. The initial condition (3) is replaced by

$$
\zeta=0, \text { at } t=0 .
$$

In this weakly nonlinear limit, the shock conditions (4) reduce to

$$
(S-\Delta)[\zeta]+\frac{3}{4}\left[\zeta^{2}\right]=0 .
$$

This can also of course be directly deduced from (5).

\section{Hydraulic flow}

\subsection{Steady solutions}

Here we consider the hydraulic theory, and to begin with we review the well-known theory (see Baines (1995) for instance) for flow over a single obstacle obstacle. Then we will show how this can be extended to obtain analogous solutions for flow over two obstacles. Thus we seek steady solutions, so that on omitting the time derivatives, equations $(1,2)$ integrate to

$$
H U=(1+\zeta-f) U=Q, \quad \zeta+\frac{U^{2}}{2}=B
$$

Here $Q, B$ are positive integration constants, representing mass flux and energy flux respectively (strictly $Q$ is volume flux, but we are assuming that the fluid density has been scaled 
to unity, and $B$ is the Bernoulli constant, while $B Q$ is the energy flux). Eliminating $H$ or $U$ gives

$$
\frac{G^{4 / 3}}{2}+\frac{1}{G^{2 / 3}}=\frac{B+1-f}{Q^{2 / 3}}, \quad G=\frac{U}{H^{1 / 2}}=\frac{U^{3 / 2}}{Q^{1 / 2}}=\frac{Q}{H^{3 / 2}} .
$$

which determines the local Froude number $G$ as a function of the obstacle height $f$.

For noncritical flow, this solution must connect smoothly to $U=F, \zeta=0$, that is $G=F$, at infinity, and so $Q=F, B=F^{2} / 2$. Noting that then the right-hand side of the first expression in (9) has a minimum value of $3 / 2-\epsilon_{m}$ when $F=1$, it can be established that

$$
0<\epsilon_{m}<1+\frac{F^{2}}{2}-\frac{3 F^{2 / 3}}{2}
$$

Here $\epsilon_{m}$ is the maximum obstacle height. This expression is plotted in figure 1 at equality (note that this is the curve BAE in figure 2.11 in Baines (1995)). It defines the subcritical regime $F<F_{b}<1$ where $F<G<1$, and the supercritical regime $1<F_{p}<F$ where $1<$ $G<F$ and a smooth steady hydraulic solution exists. In the subcritical regime a localised depression forms over the obstacle, and in the supercritical regime a localised elevation forms over the obstacle. For small $\left(\epsilon_{m}\right)^{1 / 2}<<1$, recalling that $\Delta=F-1$, we find that

$$
\Delta_{p, b}= \pm \frac{\left(6 \epsilon_{m}\right)^{1 / 2}}{2}+\frac{\epsilon_{m}}{4}+O\left(\epsilon_{m}^{3 / 2}\right) .
$$

In the transcritical regime $F_{b}<F<F_{p}$ (10) does not hold and is replaced by

$$
\epsilon_{m}>1+\frac{F^{2}}{2}-\frac{3 F^{2 / 3}}{2} \text {. }
$$

Instead we seek a solution which has upstream and downstream shocks propagating away from the obstacle, and which satisfies the critical flow condition at the top of the obstacle, that is, when $f=\epsilon_{m}, G_{x} \neq 0$. This condition implies that

$$
G=1, \quad \frac{3 Q^{2 / 3}}{2}=B+1-\epsilon_{m} \quad \text { at } \quad f=\epsilon_{m} .
$$

For a given $\epsilon_{m}$, this relation defines $B$ in terms of $Q$. At this critical location $U=U_{m}=Q^{1 / 3}$ and $1+\zeta_{m}-\epsilon_{m}=Q^{2 / 3}$. The local Froude number varies over the range $G_{-}<G<G_{+}$where \pm denote the downstream and upstream values, It transpires that in order for the shocks to propagate away from the obstacle the flow is subcritical upstream where $G_{-}<G<1$, $\zeta_{-}>\zeta>\zeta_{m}, U_{-}<U<U_{m}$, and supercritical downstream $1<G<G_{+}, \zeta_{+}<\zeta<\zeta_{m}$, $U_{+}>U>U_{m}$

Before proceeding we note that the expressions (9) hold both upstream and downstream, yielding the relationships

$$
\begin{gathered}
U_{ \pm}\left(1+\zeta_{ \pm}\right)^{1 / 2}=Q, \quad \frac{U_{ \pm}^{2}}{2}+\zeta_{ \pm}=B \\
\text { and so } \quad \frac{U_{ \pm}^{2}}{2}+\frac{Q}{U_{ \pm}}=\frac{Q^{2}}{2\left(1+\zeta_{ \pm}\right)^{2}}+\zeta_{ \pm}+1=B+1 \\
\text { while } \quad \frac{G_{ \pm}^{4 / 3}}{2}+\frac{1}{G_{ \pm}^{2 / 3}}=\frac{B+1}{Q^{2 / 3}} .
\end{gathered}
$$

For given $Q, B$, these relations fix $U_{ \pm}, \zeta_{ \pm}$completely. But we have one relationship (13) connecting $B, Q$, and so there is just a single constant to determine. This is found using the classical shock closure described in the next subsection. 


\subsection{Classical shock closure}

Outside the obstacle $U=U_{ \pm}, \zeta=\zeta_{ \pm}$are constants, downstream and upstream respectively, and are connected to the undisturbed values $U=F, \zeta=0$ far downstream and upstream, using classical shock closure based on the shock conditions (4), see figure 2. Since the steady hydraulic flow over the obstacle conserves mass and energy, rather than mass and momentum, these are nontrivial conditions to apply. Further, it transpires that we cannot simultaneously impose upstream and downstream jumps which connect directly to the uniform flow. Instead, we first impose an upstream jump as specified by Baines (1995), see also El et al. (2009). There is then a downstream jump which connects to a rarefaction wave, see figure 2 .

First we consider the upstream jump, which connects $\zeta_{-}, U_{-}$to $0, F$ with $S_{-}<0$. The first relation in (4) gives

$$
\zeta_{-}\left(S_{-}-U_{-}\right)=U_{-}-F, \quad \text { or } \quad S_{-} \zeta_{-}=Q-F,
$$

and the second relation in (4) gives

$$
\left(1+\zeta_{-}\right)\left(U_{-}-F\right)\left(S_{-}-U_{-}\right)=\zeta_{-}\left(1+\frac{\zeta_{-}}{2}\right) .
$$

Eliminating $S_{-}$, or $U_{-}-F$ yield the following expressions

$$
\begin{gathered}
\left(1+\zeta_{-}\right)\left(U_{-}-F\right)^{2}=\zeta_{-}^{2}\left(1+\frac{\zeta_{-}}{2}\right), \\
S_{-}=F-\left[\left(1+\zeta_{-}\right)\left(1+\frac{\zeta_{-}}{2}\right)\right]^{1 / 2} . \\
\text { and }\left(1+\zeta_{-}\right) F-\zeta_{-}\left[\left(1+\zeta_{-}\right)\left(1+\frac{\zeta_{-}}{2}\right)\right]^{1 / 2}=Q .
\end{gathered}
$$

Since we need $S_{-}<0$ it follows that we must have $\zeta_{-}>0$ and $U_{-}<Q<F$. The system of equations is now closed, as the combination of (15) and (21) determines $\zeta_{-}$in terms of $B$, so that finally all unknowns are obtained in terms of $\epsilon_{m}$ from (13). Further, the condition $\zeta_{-}>0$ serve to define the transcritical regime (12) in terms of the Froude number $F$ and $\epsilon_{m}$.

Downstream, this procedure also determines $U_{+}>F, \zeta_{+}<0$, but in general, this cannot be resolved by a jump directly to the state $F, 0$. Instead we must insert a right-propagating rarefaction wave, see figure 2 . The rarefaction wave propagates downstream into the undisturbed state $0, F$, and so is defined by the values $U_{r}, \zeta_{r}$ where

$$
U_{r}-2\left(1+\zeta_{r}\right)^{1 / 2}=F-2 .
$$

It is then connected to the hydraulic downstream state $U_{+}, \zeta_{+}$by a shock, using the jump conditions (4) to connect the two states through a shock with speed $S_{+}>0$. There are then three equations for the three unknowns $\zeta_{r}, U_{r}, S_{+}$and the system is closed.

In the weakly nonlinear regime, when the forcing is sufficiently small (the appropriate

small parameter is $\alpha \sim \sqrt{\epsilon_{m}}$ ), the rarefaction wave contribution can be neglected as it has the amplitude of order $\alpha^{3}$ while the shock intensity is $O(\alpha)$. In this limit we can solve the system of equations by an expansion in $\alpha$ and find that

$$
3 \zeta_{ \pm}=2 \Delta \mp\left(6 \epsilon_{m}+\beta_{ \pm}\right)^{1 / 2}+O\left(\alpha^{3}\right), \quad \beta_{ \pm}=3 \zeta_{ \pm}^{3}-2 \zeta_{ \pm} \Delta^{2}+\frac{4 \Delta^{3}}{9}
$$




$$
\begin{gathered}
S_{ \pm}=\Delta-\frac{3 \zeta_{ \pm}}{4}+\frac{\zeta_{ \pm}^{2}}{32}+O\left(\alpha^{3}\right), \quad G_{ \pm}=1+\Delta-\frac{3 \zeta_{ \pm}}{2}+\gamma_{ \pm}+O\left(\alpha^{3}\right), \quad \gamma_{ \pm}=\frac{9 \zeta_{ \pm}^{2}}{8}-\frac{\zeta_{ \pm} \Delta}{2} \\
Q=1+\Delta+\zeta_{ \pm} \Delta-\frac{3 \zeta_{ \pm}^{2}}{4}+O\left(\alpha^{3}\right) .
\end{gathered}
$$

Here $\beta_{ \pm}=O\left(\alpha^{3}\right), \gamma_{ \pm}=O\left(\alpha^{2}\right)$ are small correction terms, which if needed explicitly can be evaluated to leading order using the leading order solution for $\zeta_{ \pm}$. It is useful to note here that using (23) and (11), the local Froude numbers

$$
G_{ \pm}=1 \pm \frac{\left(6 \epsilon_{m}\right)^{1 / 2}}{2}+O\left(\alpha^{2}\right)=1+\Delta_{p, b}+O\left(\alpha^{2}\right)
$$

and are independent of $\Delta$ at the leading order in $\alpha$. Also, since the transcritical regime is defined by $\Delta_{s}<\Delta<\Delta_{p}$ it follows that at the leading order in $\alpha$, the local downstream and upstream Froude numbers $G_{ \pm}$are outside this transcritical regime, and hence the downstream and upstream flows are indeed fully supercritical and subcritical respectively.

\subsection{Two obstacles}

The same procedure can now be followed when there are two widely separated obstacles. Based on our numerical simulations reported in section 3, the solution evolves in two stages. In the first stage the theory described above can be applied to each obstacle separately. Then in the second stage when the downstream propagating waves emitted by the first obstacle interact with the upstream propagating waves emitted by the first obstacle, an interaction takes place and there is an adjustment to a new configuration. There are several scenarios depending on the obstacles heights $\epsilon_{1,2}$ and the Froude number $F$. For instance if both obstacles satisfy the condition (10) for subcritical or supercritical flow then the obtained solutions for each obstacle separately will again be obtained. On the other hand if both obstacles satisfy the condition (12) for transcritical flow then at the end of the first stage a downstream depression shock preceded by a rarefaction wave emitted by the first obstacle will meet an upstream elevation shock emitted by the second obstacle. Our numerical simulations show that these generate a new shock between the obstacles. The speed $S_{\text {int }}$ of this shock can be found from (4) where the conservation of mass law implies that

$$
S_{i n t}\left(\zeta_{2-}-\zeta_{1+}\right)=\left(1+\zeta_{2-}\right) U_{2-}-\left(1+\zeta_{1+}\right) U_{1+}+O\left(\alpha^{3}\right)=Q_{2}-Q_{1}+O\left(\alpha^{3}\right) .
$$

Here the $O\left(\alpha^{3}\right)$ error is due to the presence of the rarefaction wave. Since $\zeta_{2-}>0>\zeta_{1+}$ the shock moves in the positive or negative direction depending on whether $Q_{2}>(<) Q_{1}$. Indeed using the expressions $(24,21)$

$$
S_{i n t}=\Delta-\frac{3}{4}\left(\zeta_{1+}+\zeta_{2-}\right)+O\left(\alpha^{2}\right)=\frac{1}{4}\left(\left\{6 \epsilon_{1}\right\}^{1 / 2}-\left\{6 \epsilon_{2}\right\}^{1 / 2}\right)+O\left(\alpha^{2}\right),
$$

and is independent of $\Delta$ to this order. Thus, this shock will move towards the higher of the two obstacles, that is, $S_{\text {int }}$ is positive or negative according as $\epsilon_{1}>\epsilon_{2}$ or $\epsilon_{1}<\epsilon_{2}$ respectively. This is followed by the interaction of this shock with either the second or first obstacle, followed eventually by an adjustment to a final localised steady state encompassing both obstacles; this is the second stage.

The final localised steady hydraulic state can now be determined as before, with the criterion that criticality occurs at the higher obstacle so that the formulae in subsections 
2.1, 2.2 apply with $\epsilon_{m}=\max \left[\epsilon_{1}, \epsilon_{2}\right]$, the same as if the combination of the two obstacles was a single obstacle. Indeed, the criticality determined at the first stage at the higher obstacle persists into the second stage, while the flow at the lower obstacle adjusts in the second stage to be locally supercritical if this lower obstacle is the second obstacle, or is locally subcritical if the lower obstacle is the first obstacle.

The final localised steady hydraulic state can now be determined as before, with the criterion that criticality occurs at the higher obstacle so that the formulae in subsections 2.1, 2.2 apply with $\epsilon_{m}=\max \left[\epsilon_{1}, \epsilon_{2}\right]$, the same as if the combination of the two obstacles was a single obstacle. Indeed, the criticality determined at the first stage at the higher obstacle persists into the second stage, while the flow at the lower obstacle adjusts in the second stage to be locally subcritical if the lower obstacle is the first obstacle, or is locally supercritical if the lower obstacle is the second obstacle. Illustrative example taken from the numerical simulations are shown in figures 3 and 4 respectively. Note that criticality is controlled by the higher obstacle which has the same height in the two cases, and hence the same constant values of $Q, B$ are generated in the region containing both obstacles.

When the obstacles have equal heights, $\epsilon_{1}=\epsilon_{2}$, then also $Q_{1}=Q_{2}$ and the shock speed $S_{\text {int }}=0\left(\alpha^{3}\right)$, so that the error term in $(27)$ is needed to determine the shock speed. This error term is due to the neglected rarefaction wave, and when this has a negative mass flux as sketched in the scenario shown in figure $2, S_{\text {int }}<0$. The numerical solutions show that this is indeed the case. Hence it is then the second obstacle which controls criticality. An example taken from our numerical simulations is shown in figure 5 . In the region over both obstacles combined there is a steady state with constant values of $Q, B$ satisfying the relation (13). The local Froude number $G=1$ at the crest of the second obstacle, where $G$ passes smoothly from subcritical $G<1$ to supercritical $G>1$. The flow is subcritical over the first obstacle, but $G=1$ at the crest of the first obstacle. At this location there is a discontinuity in the slope of $G$, and hence also in the slopes of $U, H$, but all quantities are continuous This can be deduced from $(9,13)$ where near the crest of either obstacle

$$
(G-1)^{2} \approx \frac{3\left(\epsilon_{m}-f\right)}{2 Q^{2 / 3}}
$$

There are two possible solutions. We consider for simplicity the generic case when $\epsilon_{m}-f \approx$ $\delta(x \pm L)^{2}, \delta>0$. Then at the second obstacle there is a smooth solution for which $G-1 \approx$

$C(x-L), C=\sqrt{3 \delta / 2 Q^{2 / 3}}$, but at the first obstacle the solution is $1-G \approx C|x+L|$, which is continuous but has a discontinuous slope. This can be regarded as a stationary contact discontinuity. This scenario is asymmetrical and so differs from those considered by Pratt (1984) who examined only symmetrical configurations and showed these could not be stable. Further he pointed out that it is not possible to construct a steady stable solution using a stationary shock as this would then dissipate energy (see the last paragraph of his section 1 and footnote on page 1216).

\subsection{Reduced model}

Before presenting the numerical results, it is useful to examine the same scenario presented above in subsections 2.1, 2.2, 2.3 using the reduced model, especially as then the initial value problem can be solved, see Grimshaw and Smyth (1986) and Grimshaw (2010) for instance. With the initial condition that $\zeta=0$ at $t=0$, equation (5) can be solved using 
characteristics,

$$
\begin{aligned}
& \frac{d x}{d t}=\Delta-\frac{3 \zeta}{2}, \quad \frac{d \zeta}{d t}=\frac{f_{x}}{2}, \\
& x=x_{0}, \quad \zeta=0, \quad \text { at } \quad t=0 .
\end{aligned}
$$

The system (30) can be integrated to yield

$$
\begin{aligned}
& \Delta \zeta-\frac{3 \zeta^{2}}{4}=\frac{1}{2}\left(f(x)-f\left(x_{0}\right)\right), \\
& 3 \zeta=2 \Delta \mp \Delta\left\{4 \Delta^{2}+6\left[f\left(x_{0}\right)-f(x)\right]\right\}^{1 / 2} .
\end{aligned}
$$

Here the upper sign is chosen until the characteristic reaches a turning point where $2 \Delta=3 \zeta$ and then the lower sign is chosen. When $\Delta=0$ the upper (lower) sign is chosen on the left-hand (right-hand) side of the maximum point where $f=\epsilon_{m}$. Where characteristics intersect, a shock forms with speed $S$, given by (7) Then when

$$
2 \Delta^{2}<3 \epsilon_{1,2}
$$

there is a critical $x_{0 c}$ for each obstacle such that all characteristics with $x_{0}<x_{0 c}$ have a turning point, propagate upstream and form an upstream shock. Otherwise all characteristics with $x_{0}>x_{0 c}$ have no turning points, propagate downstream and form a downstream shock. The critical point is defined by $3 f\left(x_{0 c}\right)=3 \epsilon_{1,2}-2 \Delta^{2}$. Then, in the first stage, a steady solution will emerge over each obstacle, terminated by upstream and downstream shocks, determined by that characteristic emanating from $x_{0 c}$ and the corresponding steady solution is found using (31)

$$
\begin{aligned}
& 4 \Delta^{2}-12 \Delta \zeta+9 \zeta^{2}=6\left(\epsilon_{m}-f(x)\right) \\
& 3 \zeta=2 \Delta \mp \operatorname{sign}[x \mp L]\left\{6\left[\epsilon_{m}-f(x)\right]\right\}^{1 / 2} .
\end{aligned}
$$

The upstream (downstream) shock has a magnitude $\zeta_{\mp}$ where

$$
3 \zeta_{\mp}=2 \Delta \pm\left\{6 \epsilon_{m}\right\}^{1 / 2}
$$

respectively. Note that $\zeta_{+}>0, \zeta_{-}<0$ so that the upstream shock is elevation and the downstream shock is depression. The speeds of these shocks are found from (7), that is

$$
4 S_{\mp}=2 \Delta \mp\left\{6 \epsilon_{m}\right\}^{1 / 2} \text {, }
$$

and $S_{-}<0, S_{+}>0$. while the local Froude number is

$$
G=1+\Delta-3 \zeta / 2, \text { and so } G_{\mp}=1 \mp \frac{\left\{6 \epsilon_{m}\right\}^{1 / 2}}{2} .
$$

In the first stage, this local steady solution holds only for each obstacle separately. When there are two obstacles the upstream elevation shock from the obstacle will meet the downstream depression shock from the obstacle. This generates a new shock, with speed

$$
S_{\text {int }}=\Delta-\frac{3}{4}\left(\zeta_{1+}+\zeta_{2-}\right)=\frac{1}{4}\left(\left\{6 \epsilon_{1}\right\}^{1 / 2}-\left\{6 \epsilon_{2}\right\}^{1 / 2}\right),
$$

which is independent of $\Delta$, and is positive or negative according as $\epsilon_{1}>\epsilon_{2}$ or $\epsilon_{1}<\epsilon_{2}$ respectively. These results all agree with the small amplitude limits of the corresponding expressions in the preceding subsections.. 


\section{$3 \quad$ Numerical results}

\subsection{Numerical method}

The nonlinear shallow water equations $(1,2)$ can be written as

$$
\mathcal{U}_{t}+\mathcal{F}_{x}=\mathcal{G},
$$

where $\mathcal{U}, \mathcal{F}$ and $\mathcal{G}$ represent the density vector, flux vector, and source term respectively,

$$
\mathcal{U}=\left[\begin{array}{c}
H \\
U H
\end{array}\right], \quad \mathcal{F}=\left[\begin{array}{c}
U H \\
H U^{2}+H^{2} / 2
\end{array}\right], \mathcal{G}=\left[\begin{array}{c}
0 \\
-H f_{x}
\end{array}\right] .
$$

The computational domain, $0<x<x_{L}$, is discretised by uniform cell size $\Delta x$. The cell center is denoted by $x_{i}$ where $x_{i-1 / 2}$ and $x_{i+1 / 2}$ refer the left and the right cell interface, respectively.

In discretization form, equation (38) can be written as

$$
\frac{\mathcal{U}_{i}^{n+1}-\mathcal{U}_{i}^{n}}{\Delta t}+\frac{\mathcal{F}_{i+1 / 2}^{n}-\mathcal{F}_{i-1 / 2}^{n}}{\Delta x}=\mathcal{G}_{i}^{n}
$$

Superscript $n$ refers to time step level. The gradient of flux funtion is approximated by the difference of numerical fluxes at the left, $\mathcal{F}_{i-1 / 2}^{n}$, and the right, $\mathcal{F}_{i+1 / 2}^{n}$, of cell interfaces respectively. At the cell interface $i+1 / 2$,

$$
\mathcal{F}_{i+1 / 2}^{n}=\mathcal{F}\left(\mathcal{U}_{i+1 / 2-}^{n}, \mathcal{U}_{i+1 / 2+}^{n}\right) .
$$

Numerical flux at the cell interface is a function of unknown variable on the left and the right limits, and

$$
\mathcal{U}_{i+1 / 2-}^{n}=\left[\begin{array}{c}
H_{i+1 / 2-}^{n} \\
H_{i+1 / 2-}^{n} U_{i}^{n^{2}}
\end{array}\right], \quad \mathcal{U}_{i+1 / 2+}^{n}=\left[\begin{array}{c}
H_{i+1 / 2+}^{n} \\
H_{i+1 / 2+}^{n} U_{i+1}^{n^{2}}
\end{array}\right] .
$$

Applying the hydrostatic reconstruction from Audusse et al. (2004),

$$
H_{i+1 / 2-}^{n}=\max \left(0, H_{i}+f_{i}-f_{i+1 / 2}\right) \text {, and } H_{i+1 / 2+}^{n}=\max \left(0, H_{i+1}+f_{i+1}-f_{i+1 / 2}\right) .
$$

Bottom slope is now included in the reconstruction of water depth. The value of bottom height at the corresponding interface is approximated by upwind evaluation,

$$
f_{i+1 / 2}=\max \left(f_{i}, f_{i+1}\right) .
$$

To obtain a well-balanced scheme, the gradient of source term and flux difference must be balanced at steady state, Audusse et al. (2004), so equation (40) can be rewritten as,

$$
\frac{\mathcal{U}_{i}^{n+1}-\mathcal{U}_{i}^{n}}{\Delta t}+\frac{\mathcal{F}_{l}^{n}\left(\mathcal{U}_{i}^{n}, \mathcal{U}_{i+1}^{n}, f_{i}, f_{i+1}\right)-\mathcal{F}_{r}^{n}\left(\mathcal{U}_{i-1}^{n}, \mathcal{U}_{i}^{n}, f_{i-1}, f_{i}\right)}{\Delta x}=0,
$$

with modified numerical fluxes,

$$
\mathcal{F}_{l}^{n}\left(\mathcal{U}_{i}^{n}, \mathcal{U}_{i+1}^{n}, f_{i}, f_{i+1}\right)=\mathcal{F}\left(\mathcal{U}_{i+1 / 2-}^{n}, \mathcal{U}_{i+1 / 2+}^{n}\right)+\left[0, H_{i}^{n^{2}}-H_{i+1 / 2-}^{n^{2}}\right] / 2,
$$




$$
\mathcal{F}_{r}^{n}\left(\mathcal{U}_{i}^{n}, \mathcal{U}_{i+1}^{n}, f_{i}, f_{i+1}\right)=\mathcal{F}\left(\mathcal{U}_{i+1 / 2-}^{n}, \mathcal{U}_{i+1 / 2+}^{n}\right)+\left[0, H_{i+1}^{n^{2}}-H_{i+1 / 2+}^{n^{2}}\right] / 2 .
$$

In this work, we apply weighted average flux (WAF) proposed by Siviglia and Toro (2009); Toro (1992); Toro et al. (1994) to obtain the approximation of $\mathcal{F}\left(\mathcal{U}_{i+1 / 2-}^{n}, \mathcal{U}_{i+1 / 2+}^{n}\right)$. We also apply the minmod flux limiter based on the total variation diminishing (TVD) proposed by Toro (1992) in our numerical scheme to remove spurious oscillations when simulating moving shock problem.

In our simulations, we apply transmissive boundaries to allow waves to propagate outwards on both boundaries. The bottom elevation is assumed to be two Gaussian obstacles given by

$$
f(x)=\epsilon_{1} \exp \left(-\left(x-x_{a}\right)^{2} / w\right)+\epsilon_{2} \exp \left(-\left(x-x_{b}\right)^{2} / w\right),
$$

where $\epsilon_{1}$ and $\epsilon_{2}$ are the obstacle heights, $x_{a}$ and $x_{b}=x_{a}+L$ are the center locations of the first and the second obstacle respectively, and the width of each obstacle is $w=10$.

\subsection{Equal obstacle heights}

3.2.1 $\epsilon_{1}=0.1, \epsilon_{2}=0.1$

Simulations for a subcritical case $F=0.5$ are shown in figure 6. Initially, in the first stage $(t=50)$, steady depression waves are produced over each obstacle, and small transient elevation waves travel upstream from each obstacle. In the second stage $t=70$ the transient wave from the second obstacle has passed over the first obstacle and proceeded upstream. In the final stage $(t=300)$ only the steady depression waves over each obstacle are left. In this case, the Froude number is outside the transcritical regime for both obstacles, see (10) and figure 1 ,.

Simulations for a transcritical flow case $F=1$ are shown in figure 7 . In the first stage $(t=50)$ a transcritical flow is generated over each obstacle separately, consisting of an elevation shock propagating upstream connected by a steady solution to a . a depression shock propagating downstream. The depression shock from the first obstacle meets the elevation shock from the second obstacle at around $t=130$ forming a single shock, which then propagates upstream. In the second stage $(t=400)$, there is an adjustment in which a locally steady subcritical depression wave forms over the first obstacle, while a locally steady transcritical flow forms over the second obstacle. At the same time the elevation shock and depression shock outside both obstacles continue to propagate in their separate ways. As time increases $(t=1000)$, the flow over both obstacles reaches a locally steady state with criticality controlled by the second obstacle.

Next, we examine a quantitative comparison between the nonlinear shallow water simulations the theoretical results from the reduced model presented in section 3.2. From the numerical simulations shown in figure 7 over the time range $t=400$ to 1000 we find that the respective shock magnitudes and speeds, $\zeta_{+}=-0.2574, \zeta_{-}=0.2670, S_{+}=0.1880, S_{-}=$ -0.1980 . With $\epsilon_{m}=0.1$ the local Froude numbers in equation (36) are $G_{+}=1.3873, G_{-}=$ 0.6127 , while the shock magnitudes from equation (34) are $\zeta_{+}=-0.2582, \zeta_{-}=0.2582$, and the shock speeds from (35) are $S_{+}=0.1937, S_{-}=-0.1937$. These values are in reasonable agreement with the numerical determined values. Using the more exact formulas $(23,24)$ up to the $O\left(\alpha^{2}\right)$ terms leads to $\zeta_{+}=-0.2468, \zeta_{-}=0.2691$ and $S_{+}=0.1871, S_{-}=-0.1996$, which is an improvement. Note that the effective small parameter here is $\left(6 \epsilon_{m}\right)^{1 / 2}=0.7746$ and so is not small enough for the reduced model to be completely accurate. 
Simulations for a supercritical flow case $F=1.5$ are shown in Figure 8. Initially, in the first stage $(t=30)$, steady elevation waves are produced over each obstacle, and small transient depression waves travel downstream from each obstacle. At the beginning of the second stage $(t=70)$ the transient wave from the first obstacle is passing over the second obstacle and proceeded upstream. In the final stage $(t=400)$ only the steady elevation waves over each obstacle are left. In this case, the Froude number is outside the transcritical regime for both obstacles, see (10) and figure 1 ,.

It should be noted that in the reduced model the local Froude number (36) satisfies $0.6127<G<1.3873$ for $\epsilon_{m}=0.1$. This prediction is consistent with the nonlinear simulations shown in figure 6 for subcritical flow, figure 7 for transcritical flow, and figure 8 for supercritical flow.

\subsection{2 $\epsilon_{1}=0.2, \epsilon_{2}=0.2$}

Four simulations for $F=0.5,1.0,1.5,2.0$ are shown in figures $9-12$. When $\epsilon_{m}=0.2$ transcritical flow occur in the range of $0.48<F<1.56$, see (10) and figure 1 . The reduced model predicts transcritical flow when $0.45<F<1.55$, see (11). Thus the flow is slightly transcritical for $F=0.5,1.5$, respectively nearly subcritical or supercritical, while it is transcritical for $F=1.0$, and supercritical for $F=2.0$. In all cases we expect the reduced model to provide quite good intepretation.

The nearly subcritical case shown in figure 9 can be compared with the subcritical case shown in figure 6 for $\epsilon_{1}=\epsilon_{2}=0.1$. Although the first stage $t=30,60$ is similar there is now visible two small rarefaction waves propagating to the left, and in the second stage $(t=130,800)$ a pronounced asymmetry develops with a larger depression wave over the second obstacle. This is due to this case being in the transcritical regime, and hence the second obstacle controls criticality.

The transcritical case shown in figure 10 is qualitatively similar to that in figure 7 for $\epsilon=0.1, \epsilon=0.1$. From the numerical simulations shown in figure 7 over the time range $t=40$ to 800 we find that the respective shock magnitudes and speeds, $\zeta_{+}=-0.3600, \zeta_{-}=$ $0.3810, S_{+}=0.2535, S_{-}=-0.2814$. With $\epsilon_{m}=0.2$ the local Froude numbers in equation (36) are $G_{+}=1.5477, G_{-}=0.4523$, while the shock magnitudes from equation (34) are $\zeta_{+}=-0.3651, \zeta_{-}=0.3651$, and the shock speeds from $(35)$ are $S_{+}=0.2739, S_{-}=-0.2739$. These values are in reasonable agreement with the numerical determined values. Using the more exact formulas $(23,24)$ up to the $O\left(\alpha^{2}\right)$ terms leads to $\zeta_{+}=-0.3422, \zeta_{-}=0.3867$ and $S_{+}=0.2603, S_{-}=-0.2853$, which is overall some improvement. But note here that the effective small parameter is $\left(6 \epsilon_{m}\right)^{1 / 2}=1.0954$ and can hardly be considered small.

The nearly supercritical case shown in figure 11 can be compared with the supercritical case shown in figure 8 for $\epsilon_{1}=\epsilon_{2}=0.1$. Although the first stage $(t=300)$ is rather similar there is already an asymmetry in that the elevation wave other the second obstacle is already slightly smaller than that over the first obstacle, indication that the adjustment process to the second date is beginning. This adjustment continues at $t=300$ and the final locally steady state is achieved at $t=660,1200$, in which there is criticality controlled by the second obstacle, and a locally subcritical flow over the first obstacle.

The fully supercritical case is shown in figure 12 and can also be compared with the supercritical case shown in figure 8 for $\epsilon_{1}=\epsilon_{2}=0.1$. It is quite similar although the time then to reach the second stage is much shorter. 


\subsection{Unequal obstacle heights}

3.3.1 $\epsilon_{1}=0.01, \epsilon_{2}=0.02$, and $\epsilon_{1}=0.1, \epsilon_{2}=0.2$

A transcritical case $(F=1)$ when the second obstacle is larger is shown in figure 13 for quite small amplitudes. At the first stage $(t=50)$, each obstacle generates elevation and depression shocks that can be described by the single obstacle theory. As time increases $(t=460)$ the depression shock from the first obstacle interacts with the upstream elevation shock generated by the second obstacle. A new shock is formed, called an intermediate shock as described in the analysis of section 2. Since the second obstacle is larger, the intermediate shock travels upstream and pasts over the first obstacle, leaving a locally steady depression wave in a locally subcritical flow $(t=1000)$. The speed of intermediate shock is greater than the speed of the travelling elevation shock from the first obstacle. These two shocks merge and finally form a new shock moving further upstream $(t=1800)$.

Next, we compare quantitatively these nonlinear simulations with theoretical results of from section 2. For $\epsilon_{1}=0.01$, we find from the nonlinear simulations that the upstream shock magnitude and speed are $\zeta_{-}=0.0822$, and $S_{-}=-0.0615$, while the reduced model predicts that $\zeta_{-}=0.0816$ and $S_{-}=-0.0612$, and using the more exact formulae $(23,24)$ leads to $\zeta_{-}=0.0828$ and $S_{-}=-0.0619$. Similarly, for the second obstacle with $\epsilon_{2}=0.02$, the downstream shock magnitude and speed from the simulations are $\zeta_{+}=0.1134$, and $S_{+}=0.0847$ while the reduced model predicts that $\zeta_{+}=-0.1155$ and $S_{+}=0.0866$, and using the more exact formulae $(23,24)$ leads to $\zeta_{+}=0.1132$, and $S_{+}=0.0853$. These comparisons show very good agreement for these small amplitude obstacles. Further, the intermediate shock speed from the simulation is $S_{i n t}=-0.0262$, while the theoretical expression (37) yields $S_{\text {int }}=-0.0254$. Also, note that for the nonlinear simulations when $t=1000-1800$, the two upstream elevation shocks merge to form a new one with the new speed $S_{-}=-0.0867$ which is nearly the addition of $S_{\text {int }}$ and $S_{-}$(for $\epsilon_{1}=0.01$ ).

A case with higher obstacle amplitudes, $\epsilon_{1}=0.1, \epsilon_{2}=0.2$ is shown in figure 14. The flow behaviour is quite similar to the smaller amplitude case. Here the intermediate shock speed from the simulation is $S_{i n t}=-0.1286$, but from equation (37), $S_{i n t}=-0.0802$. The quite large difference is due to higher order nonlinear effects.

\subsection{2 $\epsilon_{1}=0.02, \epsilon_{2}=0.01$, and $\epsilon_{1}=0.2, \epsilon_{2}=0.1$}

A transcritical case $(F=1)$ when the first obstacle is larger is shown in figure 15 for quite small amplitudes. At the first stage $(t=150)$, each obstacle generates elevation and depression shocks that can be described by the single obstacle theory. As time increases $(t=$ 460), the downstream depression shock from the first obstacle interacts with the upstream elevation shock generated by the second obstacle, and an intermediate shock is formed. Since the first obstacle is larger, it now controls criticality. The intermediate shock travels downstream and passes over the second obstacle, leaving a locally steady elevation wave $(t=1400)$ in a locally supercritical flow. The speed of intermediate shock is greater than the speed of the downstream travelling depression shock from the second obstacle. These two shocks merge and form a new shock moving further downstream $(t=1800)$.

Next, we compare quantitatively these nonlinear simulations with the theoretical results. For $\epsilon_{1}=0.02$, we find from the nonlinear simulations that the upstream shock magnitude and speed are $\zeta_{-}=0.1170$, and $S_{-}=-0.0880$, while the reduced model predicts that $\zeta_{-}=0.1155$ and $S_{-}=-0.0866$, and using the more exact formulae $(23,24)$ leads to $\zeta_{-}=$ 
0.1165, $S_{-}=-0.0870$. Similarly, for the second obstacle with $\epsilon_{2}=0.01$, the downstream shock magnitude and speed from the simulations are $\zeta_{+}=-0.0811$, and $S_{+}=0.0607$, while the reduced model predicts that $\zeta_{+}=-0.0816$, and $S_{+}=0.0612$, and using the more exact formulae $(23,24)$ leads to $\zeta_{-}=0.0802, S_{-}=-0.0604$. These comparisons show very good agreement for small amplitude obstacles. Further, the intermediate shock speed from the simulation is $S_{i n t}=0.0260$, while the theoretical expression (37) yields $S_{\text {int }}=0.0254$. Also, note that for the nonlinear simulations when $t=1400-1800$, the two downstream depression shocks merge to form a new shock with the new speed $S_{+}=0.0812$, which is nearly the addition of $S_{\text {int }}$ and $S_{+}$(for $\epsilon=0.01$ ).

A case with higher obstacle amplitudes, $\epsilon=0.2, \epsilon_{2}=0.1$ is shown in figure 16 . The flow behaviour is similar to the smaller amplitude case. Here the intermediate shock speed from

the simulation is $S_{i n t}=0.0281$, but from equation $(37), S_{i n t}=0.0802$. Again, the quite large difference is due to higher order nonlinear effects.

\section{Summary}

Transcritical shallow-water flow over two localised and widely-spaced obstacles has been examined using the fully nonlinear shallow water equations $(1,2)$ and with a combination of numerical simulations and theoretical analysis based on hydraulic flow concepts. For a single obstacle, the solution is typically a locally steady hydraulic flow over the obstacle contained between an upstream elevation shock and a downstream depression shock. For the case of two obstacles there are two stages. At the first stage, each obstacle generates an upstream propagating elevation shock and a downstream propagating depression shock, each well described by the single obstacle theory. Then in the second stage the downstream propagating depression shock from the first obstacle interacts with the upstream propagating elevation shock from the second obstacle to produce an intermediate shock, which propagates towards the larger obstacle, or if the obstacles have equal heights, towards the second obstacle. There is an adjustment to a locally steady flow over both obstacles where the higher obstacle obstacle controls criticality, or if the obstacles have equal heights, the second obstacle controls criticality This outcome agrees with the analytical theory based on hydraulic flow concepts extended here form a single obstacle to two obstacles.

As is known, the case of flow over a single negative obstacle, or hole, is more complicated, as the shock waves are generated at the obstacle location, see Grimshaw and Smyth (1986), Grimshaw et al. (2007) and Grimshaw et al. (2009). Hence we expect the case when either or both of the obstacles are holes could lead to different and more complicated scenarios, which will be the subject of a future study. Further the present study is restricted to non-dispersive waves and extensions to include even just weak dispersion using the forced KdV equation, or the fully nonlinear Su-Gardner equations, as done by El et al. (2009) for a single obstacle, will certainly lead to rather different behaviour. In that case, the shocks are replaced by undular bores and the shock interactions described here are replaced by the interactions of these nonlinear wave trains. For instance some of the numerical simulations reported by Grimshaw et al. (2009) using just the forced KdV equation indicate that the interaction of these nonlinear wave trains can produce very complicated behaviour. This also is a topic needing much further study. 


\section{Acknowledgements}

This work was supported by Thailand Research Fund (TRF) under the grant no. RSA5680038 to the second author.

\section{References}

Akylas, T. R. (1984). On the excitation of long nonlinear water waves by moving pressure distribution. J. Fluid Mech., 141:455-466.

Audusse, E., Bouchut, F., Bristeau, M.-O., Klein, R., and Perthame, B. (2004). A fast and stable well-balanced scheme with hydrostatic reconstruction for shallow water flows. SIAM J. Sci. Com., 25:20502065.

Baines, P. (1995). Topographic effects in stratified flows. CUP.

Binder, B., Dias, F., and Vanden-Broeck, J.-M. (2006). Steady free-surface flow past an uneven channel bottom. Theor. Comp. Fluid Dyn., 20:125-144.

Cole, S. L. (1985). Transient waves produced by flow past a bump. Wave Motion, 7:579-587.

Dias, F. and Vanden-Broeck, J. M. (2004). Trapped waves between submerged obstacles. J. Fluid Mech., 509:93-102.

Ee, B. K., Grimshaw, R. H. J., Chow, K. W., and Zhang, D.-H. (2011). Steady transcritical flow over a hole: Parametric map of solutions of the forced extended Korteweg-de Vries equation. Phys. Fluids, 23:04662.

Ee, B. K., Grimshaw, R. H. J., Zhang, D.-H., and Chow, K. W. (2010). Steady transcritical flow over an obstacle: Parametric map of solutions of the forced Korteweg-de Vries equation. Phys. Fluids, 22:056602.

El, G., Grimshaw, R., and Smyth, N. (2006). Unsteady undular bores in fully nonlinear shallow-water theory. Phys. Fluids, 18:027214.

El, G., Grimshaw, R., and Smyth, N. (2008). Asymptotic description of solitary wave trains in fully nonlinear shallow-water theory. Physica D, 237:2423-2435.

El, G., Grimshaw, R., and Smyth, N. (2009). Transcritical shallow-water flow past topography: finite-amplitude theory. J. Fluid Mech., 640:187-214.

Grimshaw, R. (2010). Transcritical flow past an obstacle. ANZIAM J., 52:1-25.

Grimshaw, R. and Smyth, N. (1986). Resonant flow of a stratified fluid over topography. $J$. Fluid Mech., 169:429-464.

Grimshaw, R., Zhang, D., and Chow, K. (2007). Generation of solitary waves by trancritical flow over a step. J. Fluid Mech., 587:235-354.

Grimshaw, R., Zhang, D.-H., and Chow, K. W. (2009). Transcritical flow over a hole. Stud. Appl. Math., 122:235-248. 
Lee, S.-J., Yates, G., and Wu, T.-Y. (1989). Experiments and analyses of upstream-advancing solitary waves generated by moving disturbances. J. Fluid Mech., 199:569-593.

Pratt, L. J. (1984). On nonlinear flow with multiple obstructions. J. Atmos. Sci., 41:12141225.

Siviglia, A. and Toro, E. (2009). WAF method and splitting procedure for simulating hydroand thermal-peaking waves in open-channel flows. J. Hydraul. Eng., 135:651-662.

Toro, E. (1992). Riemann problems and the WAF method for solving two-dimensional shallow water equations. Philos. Trans. R. Soc. London Ser. A, 338:43-68.

Toro, E., Spruce, M., and Speares, W. (1994). Restoration of the contact surface in the HLL-Riemann solver. Shock wave, 4:25-34. 


\section{Appendix}

The weakly nonlinear model (5) for small-amplitude topographic forcing in the transcritical regime can be derived as follows. First, we introduce the Riemann variables

$$
R=U+2 C, \quad L=U-2 C, \quad C=\sqrt{H},
$$

so that equations $(1,2)$ become

$$
R_{t}+(U+C) R_{x}+f_{x}=0, \quad L_{t}+(U-C) L_{x}+f_{x}=0 .
$$

Then we assume that $f \sim \alpha^{2}$ where $\alpha \ll 1$, and that $\zeta \sim \alpha, \zeta_{t} \sim \alpha^{2}, u=U-F \sim \alpha$, and $\Delta=F-1 \sim \alpha$. Next, noting that $U+C=F+1+O(\alpha)$, we can find an approximation to the right-going Riemann invariant in the vicinity of the topography,

$$
R=F+2-\frac{f}{2}+O\left(\alpha^{3}\right), \quad \text { so that } \quad u+\zeta=\frac{\zeta^{2}}{4}+\frac{f}{2}+O\left(\alpha^{3}\right) .
$$

Here a transient propagating rapidly with a speed $F+1+O(\alpha)$ to the right is ignored. Then we find that for the left-going Riemann invariant,

$$
\begin{aligned}
& L=2 U-(F+2)+\frac{f}{2}+O\left(\alpha^{3}\right)=F-2-2 \zeta+\frac{\zeta^{2}}{2}+\frac{3 f}{2}+O\left(\alpha^{3}\right), \\
& U-C=\frac{3 U}{2}-\frac{F+2}{2}+\frac{f}{4}+O\left(\alpha^{3}\right)=\Delta-\frac{3 \zeta}{2}+O\left(\alpha^{2}\right) .
\end{aligned}
$$

Thus finally the equation for $L$ in (44) reduces to (5), with an error of $O\left(\alpha^{3}\right)$. Similarly the mass shock condition in (4) reduces to (7) with an error of $O\left(\alpha^{3}\right)$, while the momentum shock condition has all terms of $O\left(\alpha^{3}\right)$. 


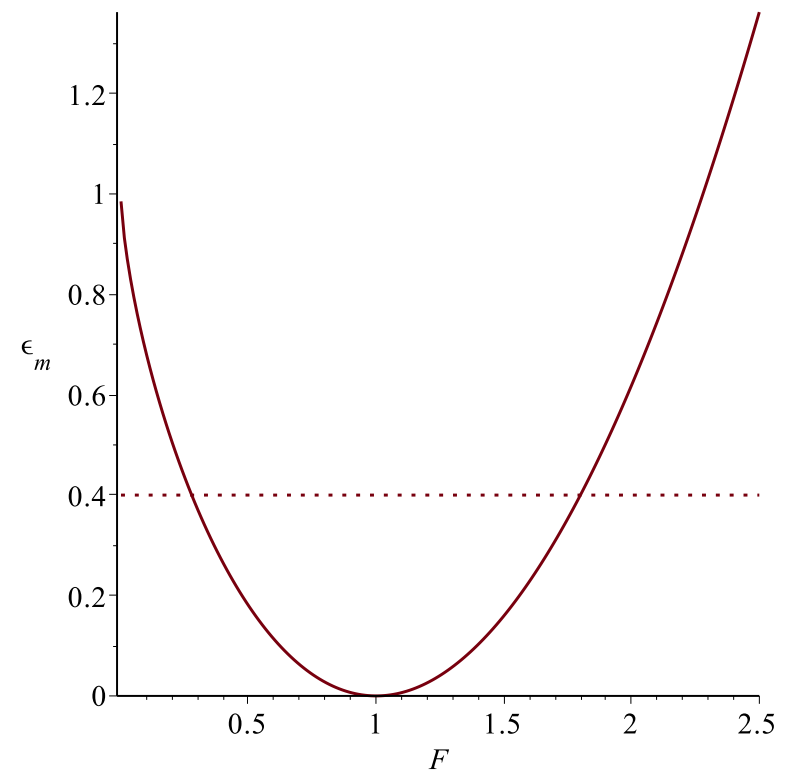

Figure 1: Plot of (10) at equality. The intersection of the line $\epsilon_{m}=$ constant with the curve (10) defines $F_{b, p}$ respectively. The region below the curve defines the subcritical and supercritical regimes, and the region above the curve is the transcritical regime.

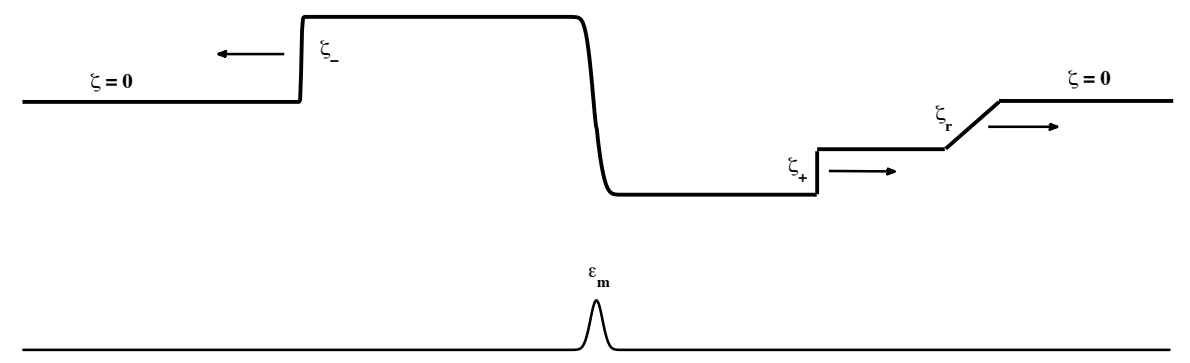

Figure 2: Schematic for closure using classical shocks. 

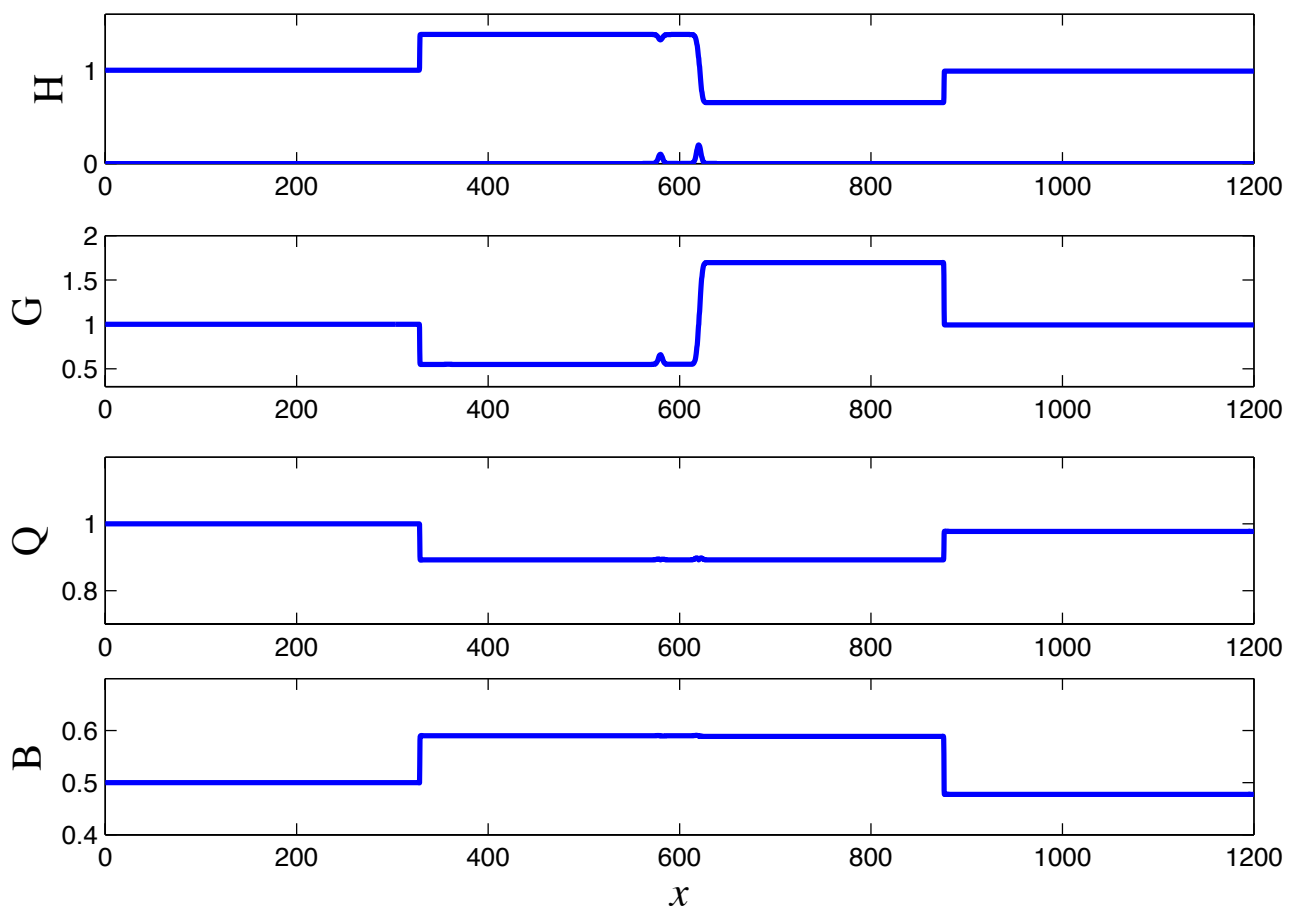

Figure 3: Hydraulic solution for the case $F=1$ and unequal obstacle heights $\epsilon_{1}=0.1, \epsilon_{2}=$ 0.2 . In the steady region over both obstacles $Q=0.8923$ and $B=0.5900$, and $G=0.6584$ at the crest of the first obstacle where the flow is locally subcritical. 

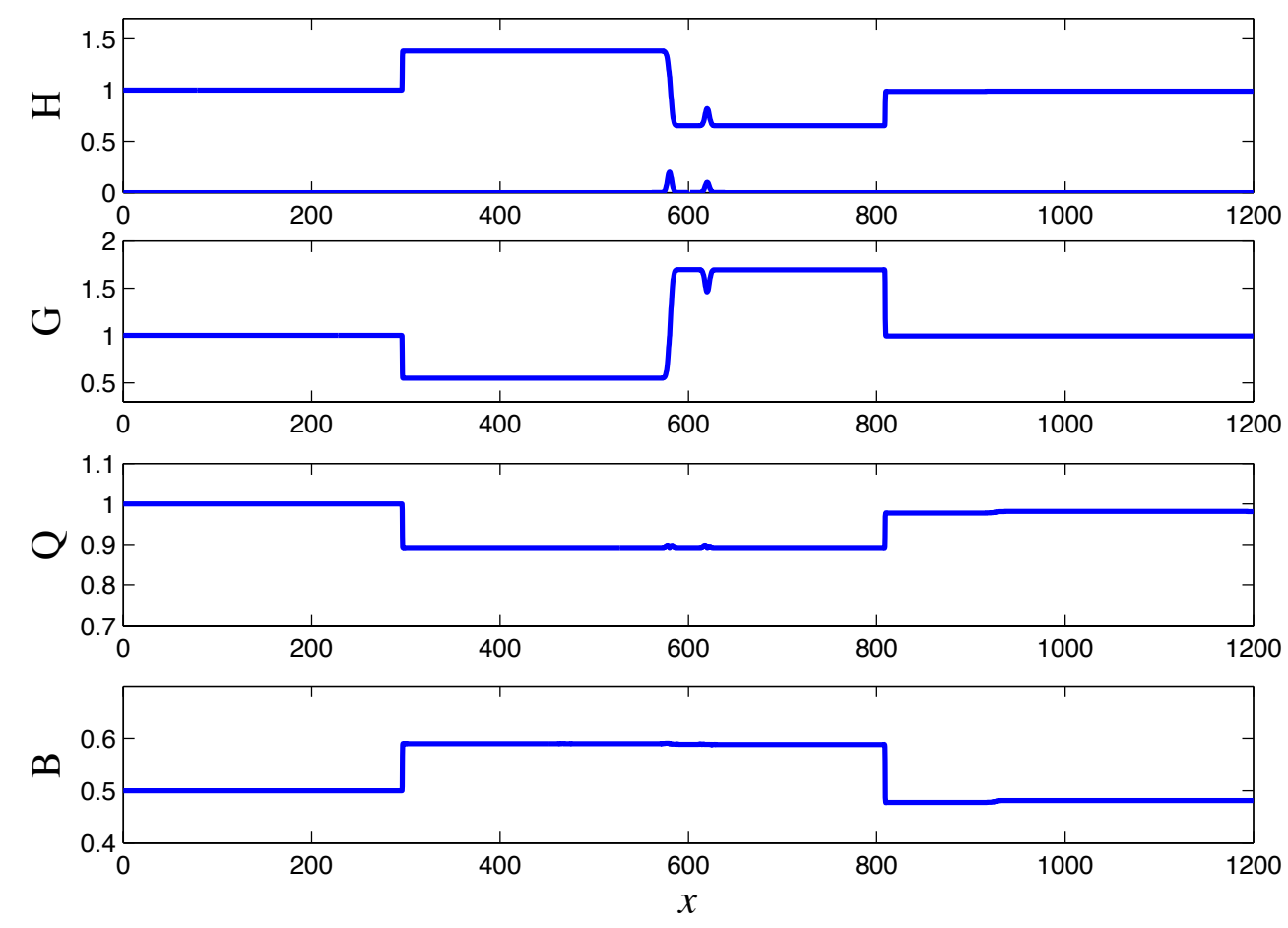

Figure 4: Hydraulic solution for the case $F=1$ and unequal obstacle heights $\epsilon_{1}=0.2, \epsilon_{2}=$ 0.1 . In the steady region over both obstacles $Q=0.8923$ and $B=0.5900$, and $G=1.463$ at the crest of the second obstacle where the flow is locally supercritical. 

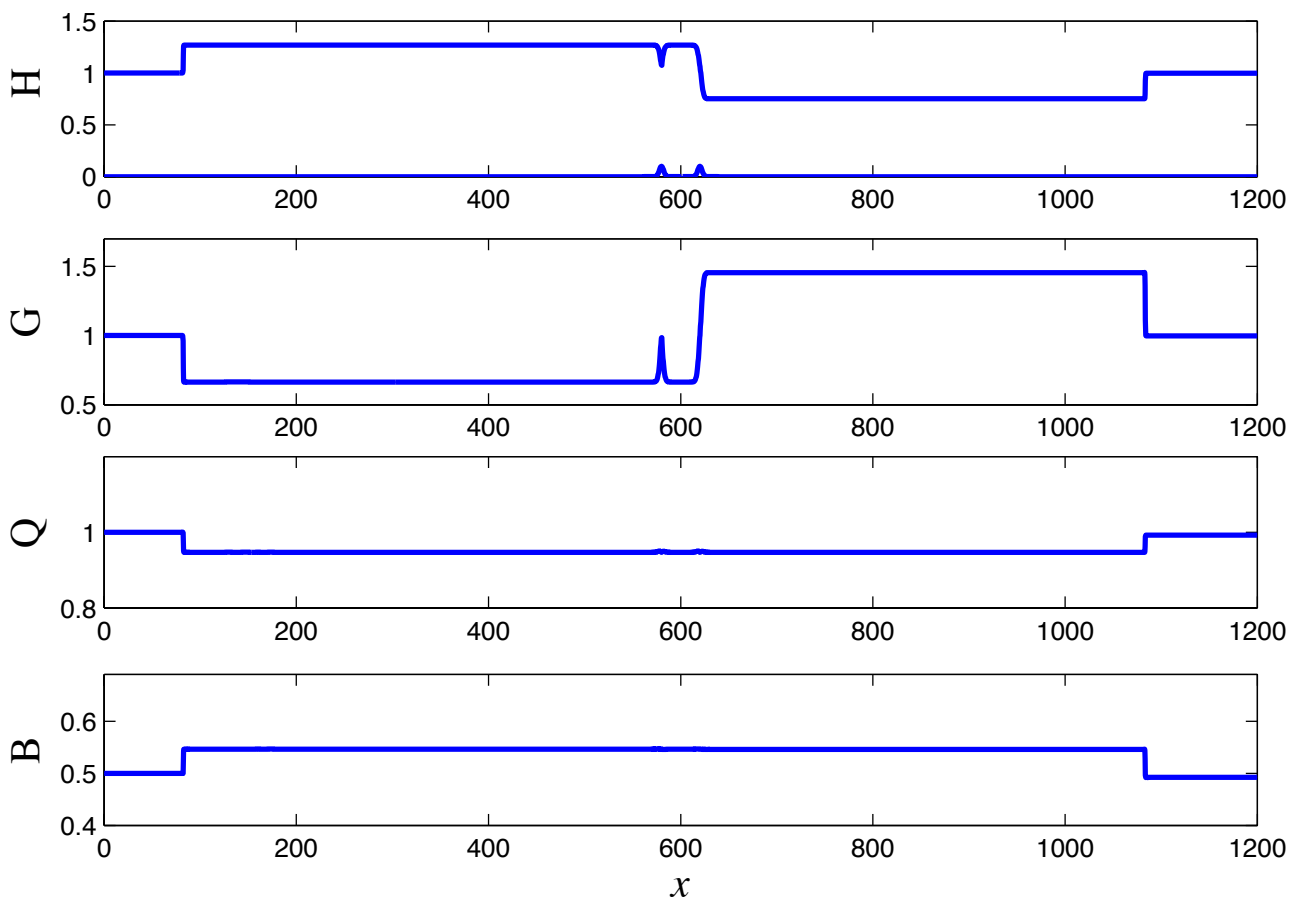

Figure 5: Hydraulic solution for the case $F=1$ and equal obstacle heights $\epsilon_{1}=\epsilon_{2}=0.1 \mathrm{In}$ the steady region over both obstacles $Q=0.9469$ and $B=0.5464$, and $G=1$ at the crest of the first obstacle, but $G<1$ in the vicinity of the first obstacle where the flow is locally subcritical. 

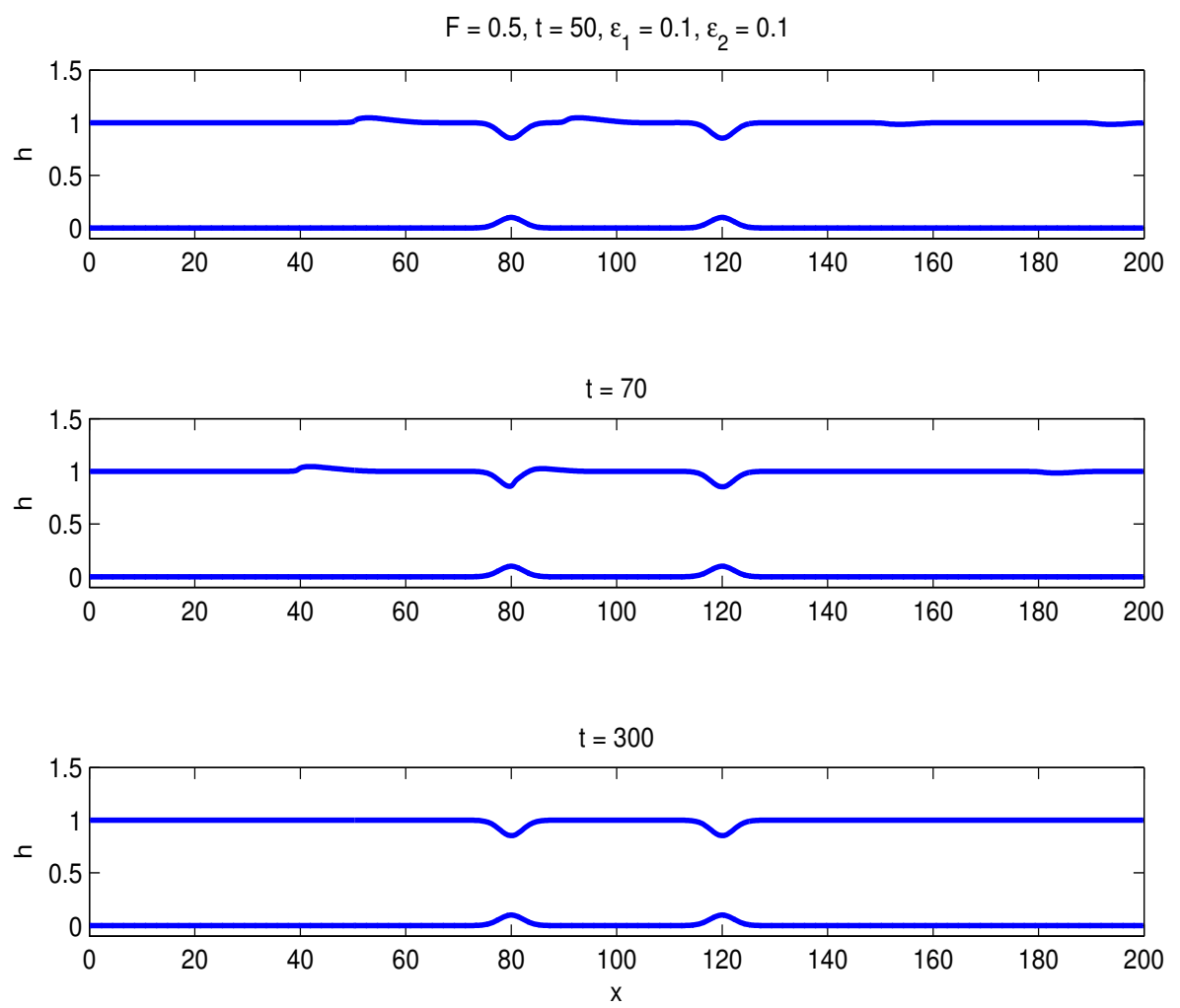

Figure 6: Simulations for $F=0.5, \epsilon_{1}=0.1, \epsilon_{2}=0.1$. 

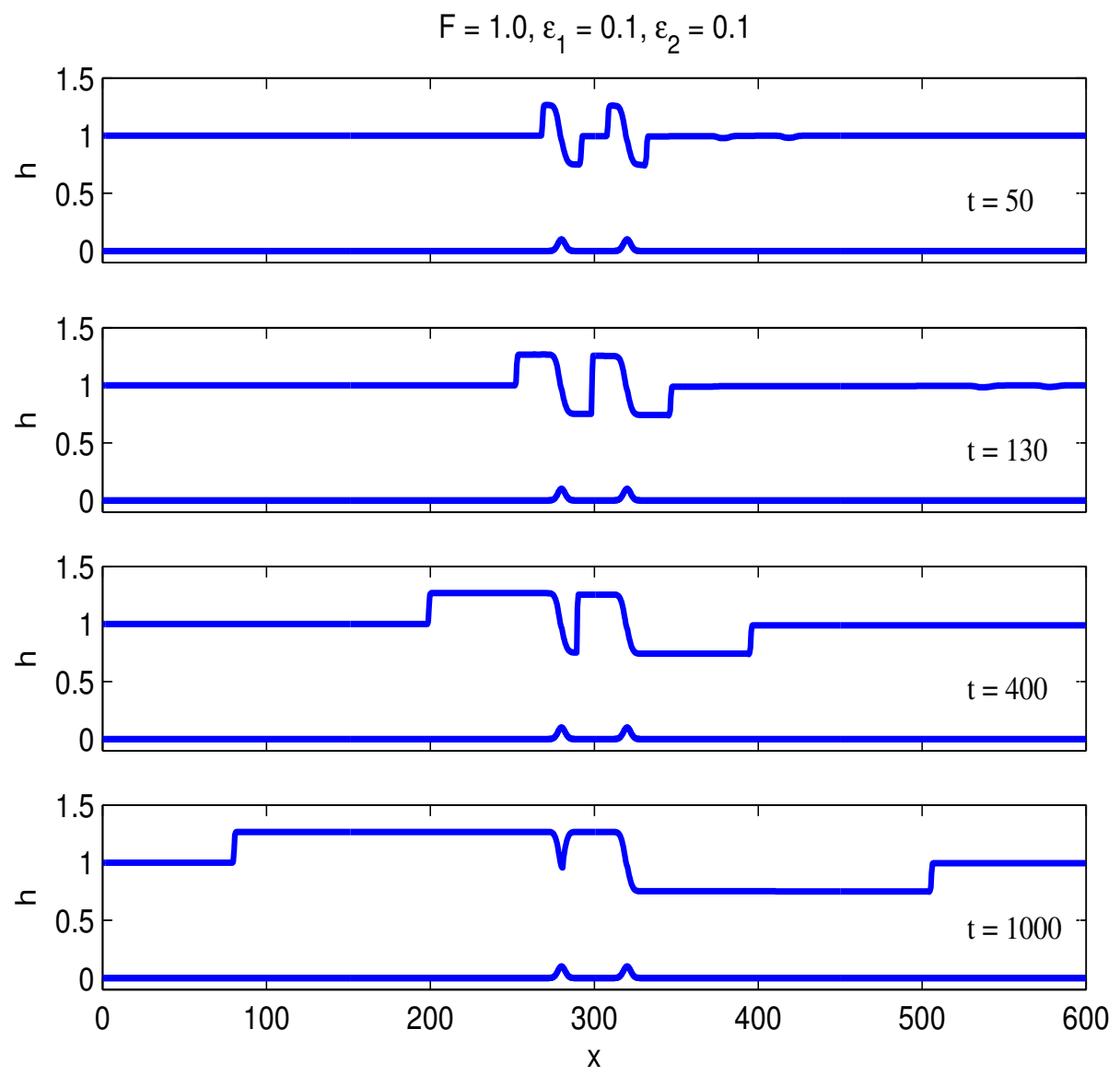

Figure 7: Simulations for $F=1.0, \epsilon_{1}=0.1, \epsilon_{2}=0.1$. 

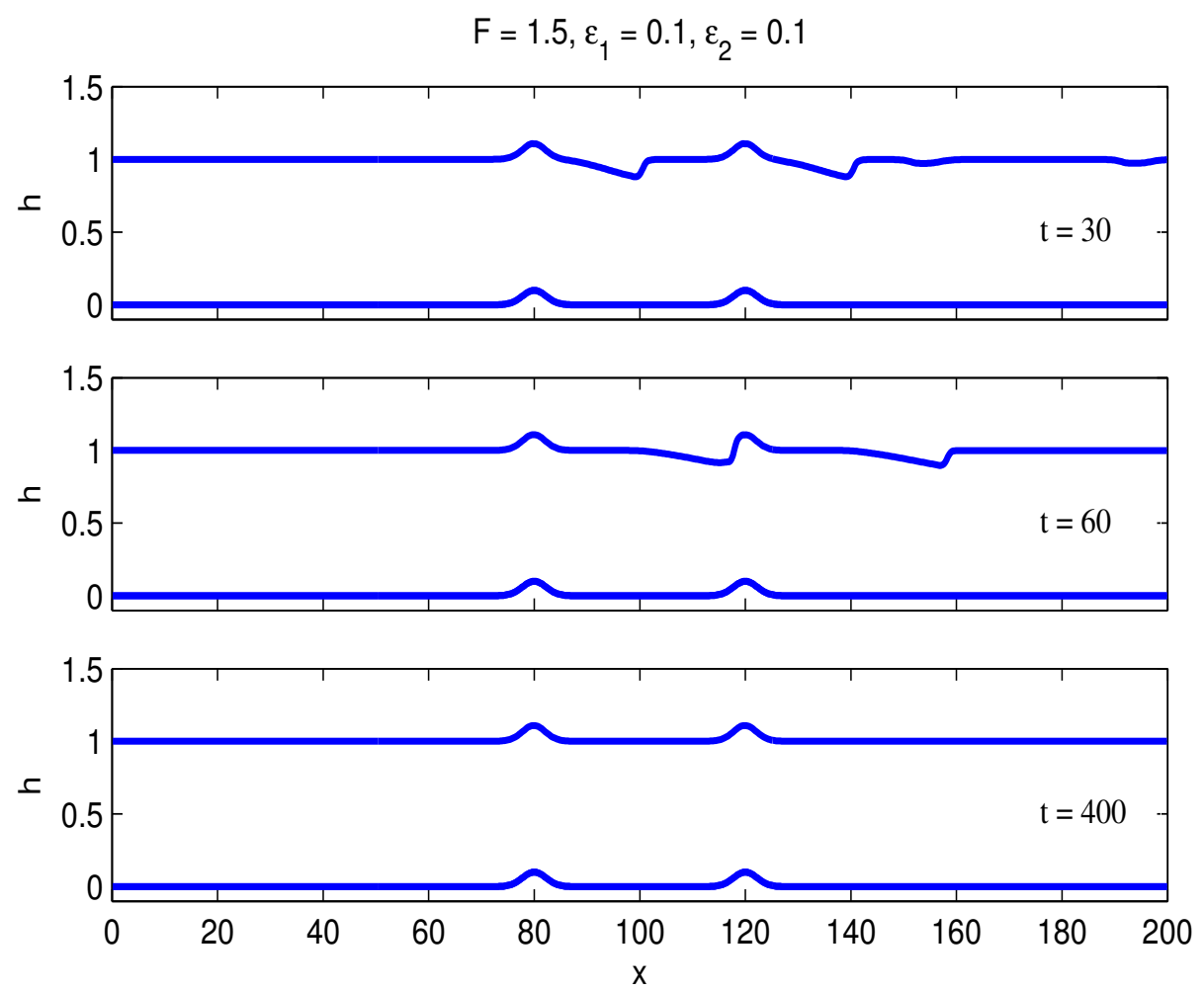

Figure 8: Simulations for $F=1.5, \epsilon_{1}=0.1, \epsilon_{2}=0.1$. 

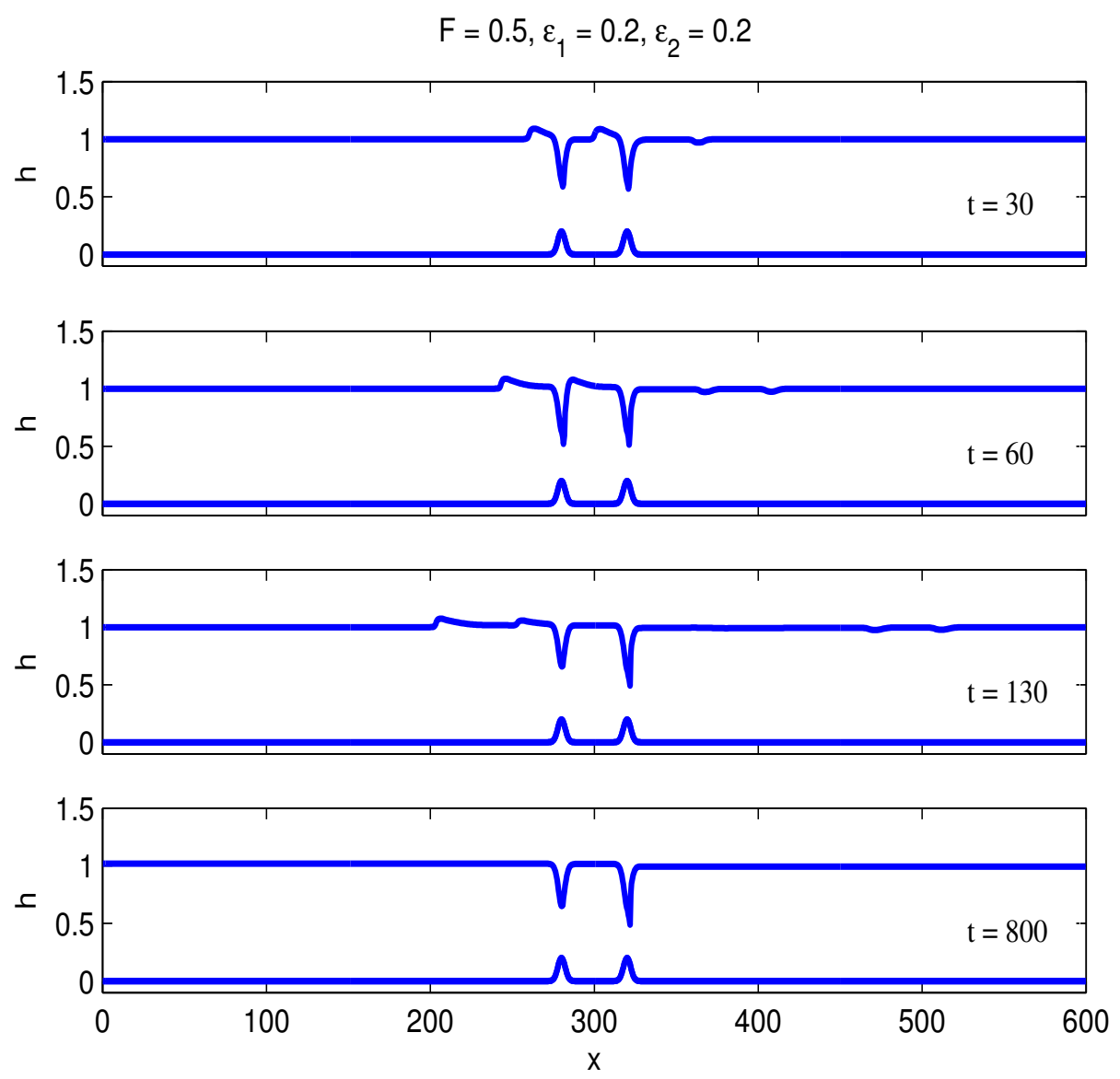

Figure 9: Simulations for $F=0.5, \epsilon_{1}=0.2, \epsilon_{2}=0.2$. 

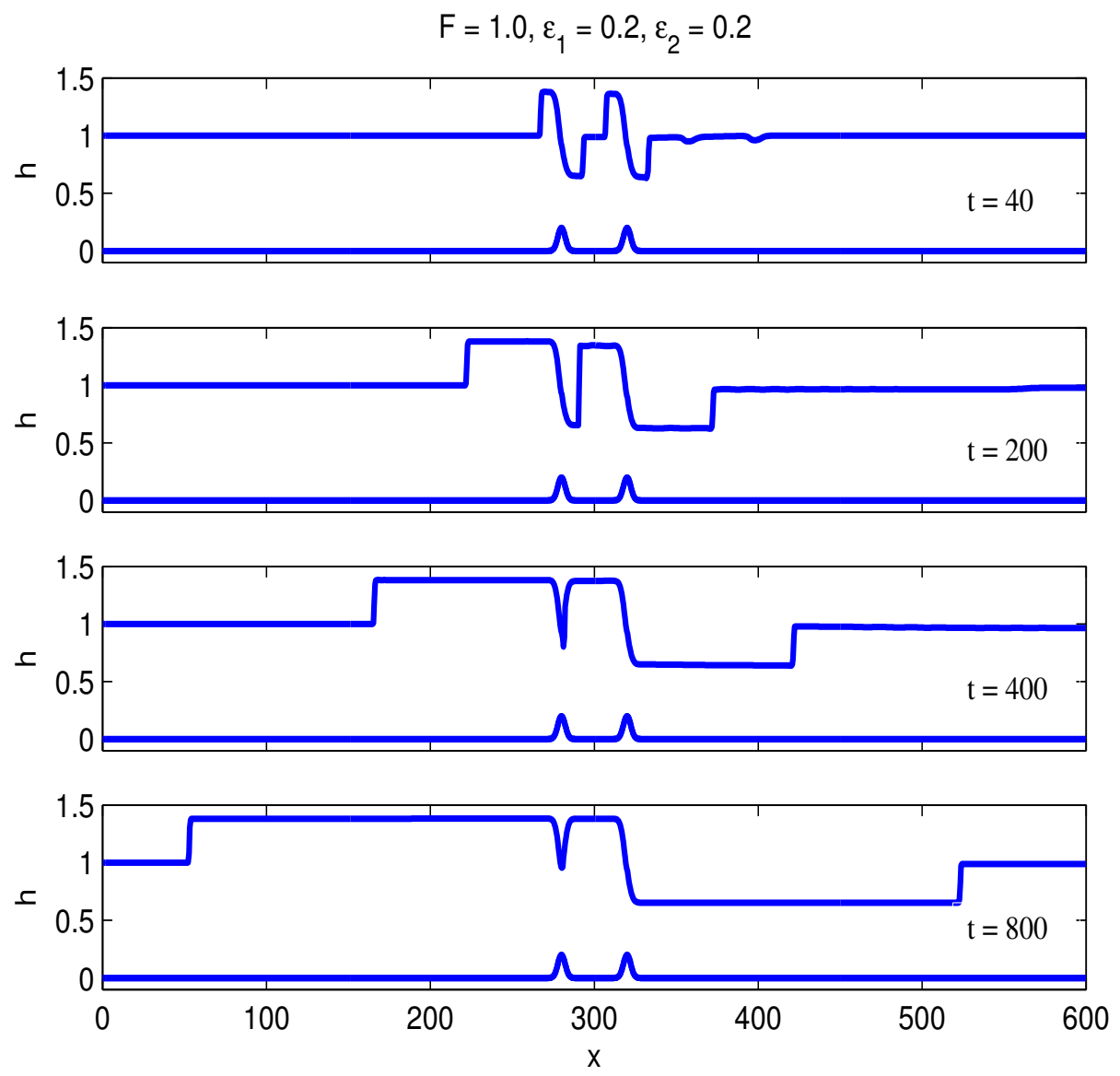

Figure 10: Simulations for $F=1.0, \epsilon_{1}=0.2, \epsilon_{2}=0.2$. 

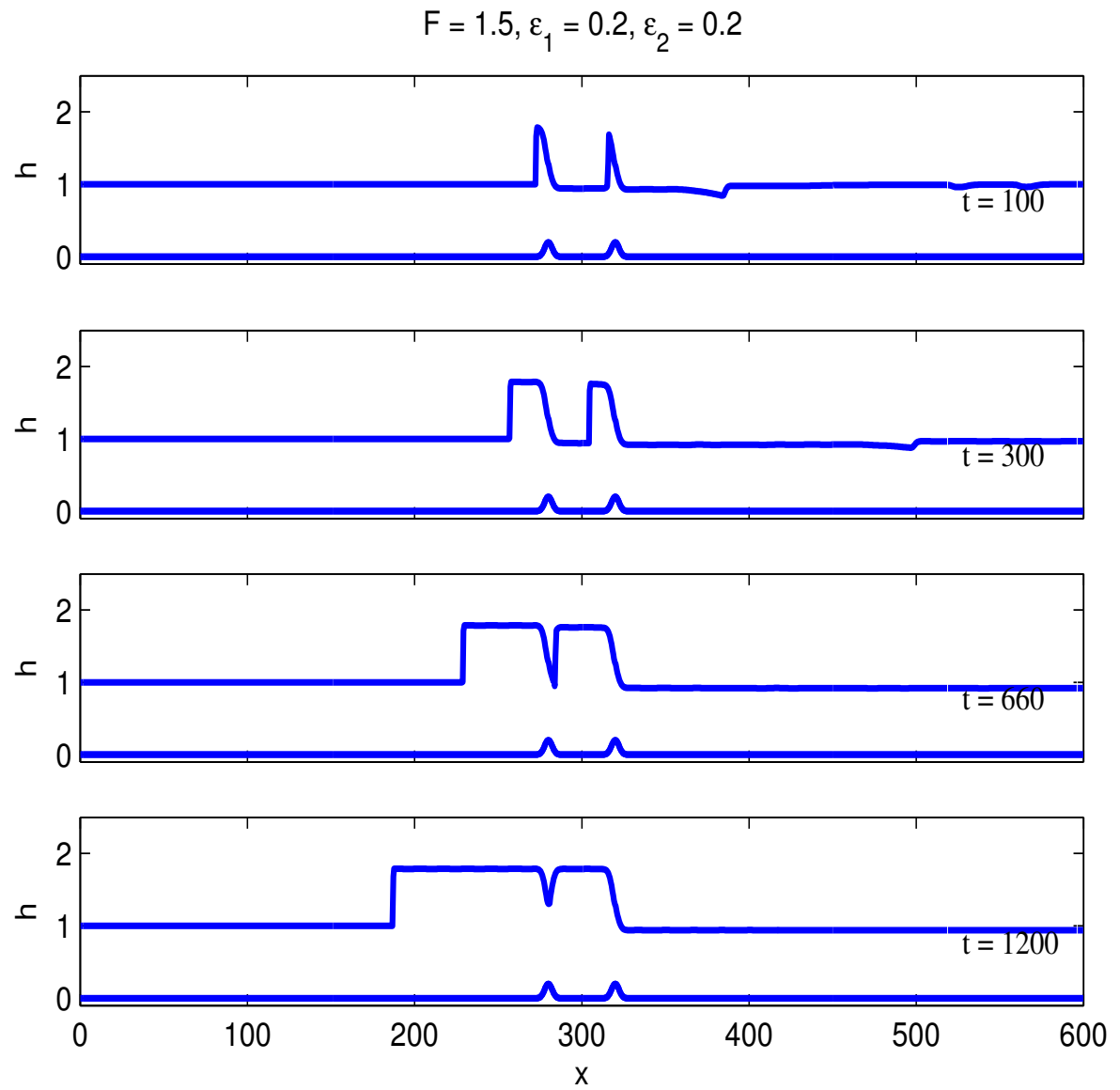

Figure 11: Simulations for $F=1.5, \epsilon_{1}=0.2, \epsilon_{2}=0.2$. 

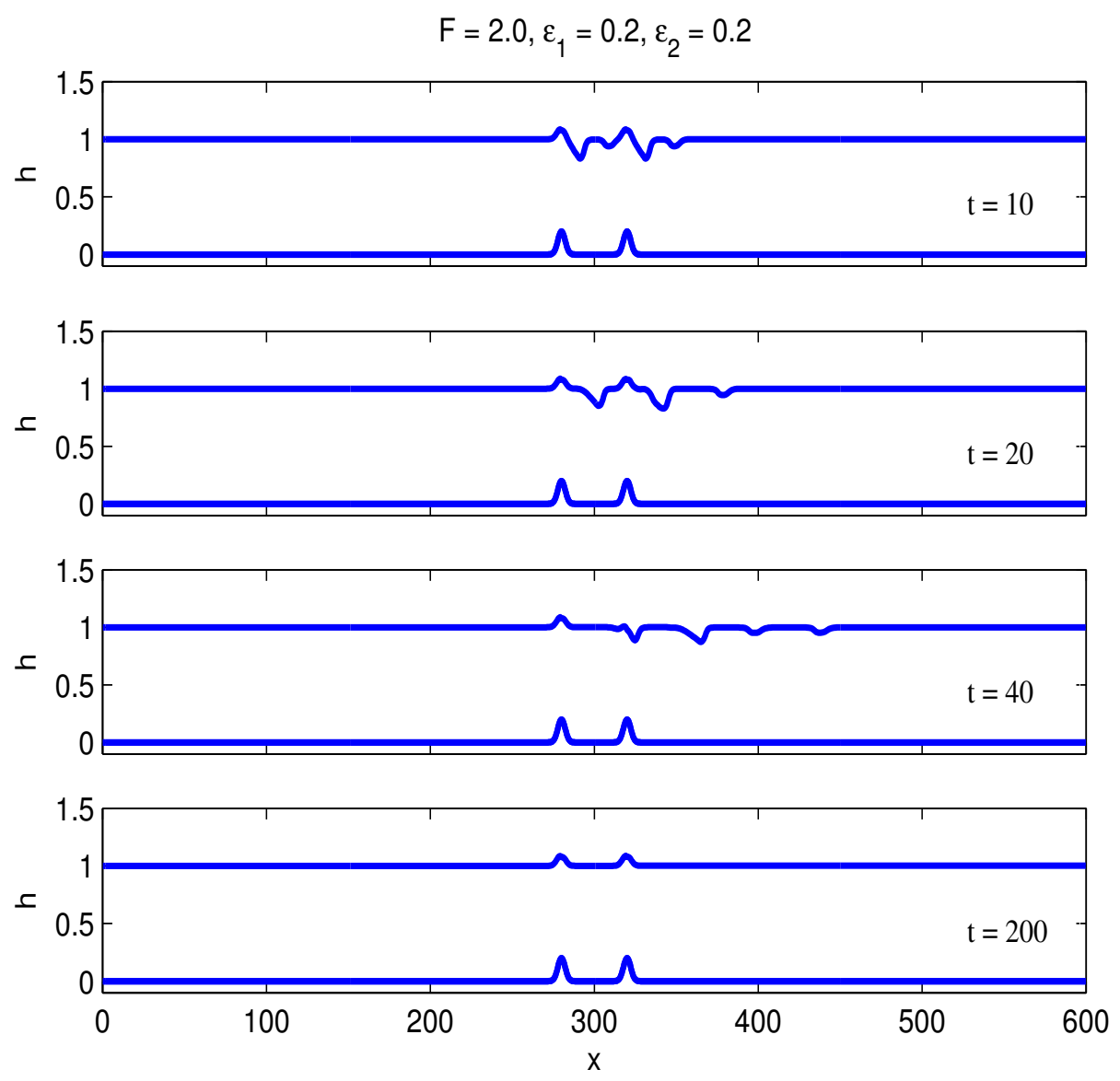

Figure 12: Simulations for $F=2.0, \epsilon_{1}=0.2, \epsilon_{2}=0.2$. 

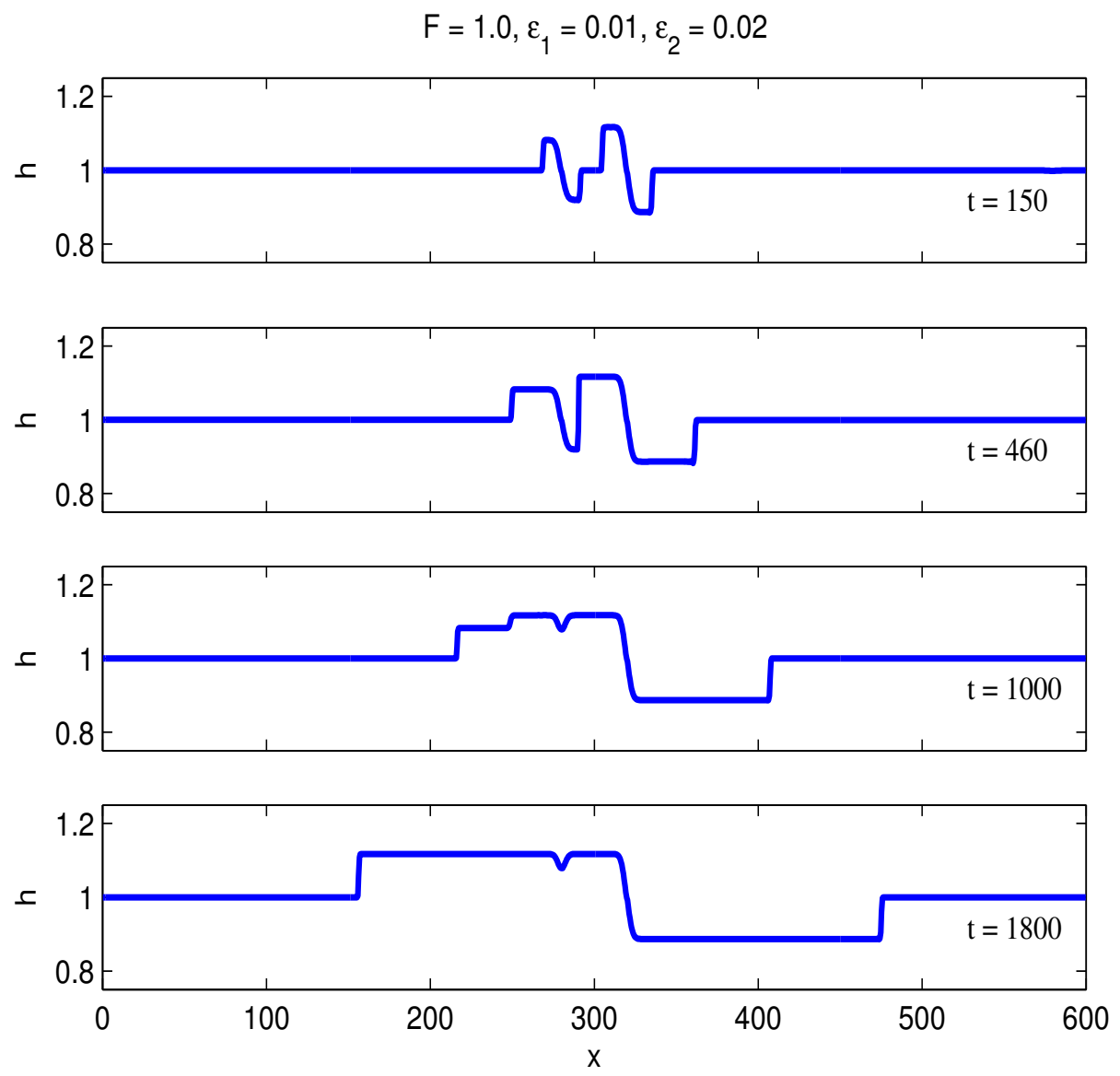

Figure 13: Simulations for $F=1.0, \epsilon_{1}=0.01, \epsilon_{2}=0.02$. 

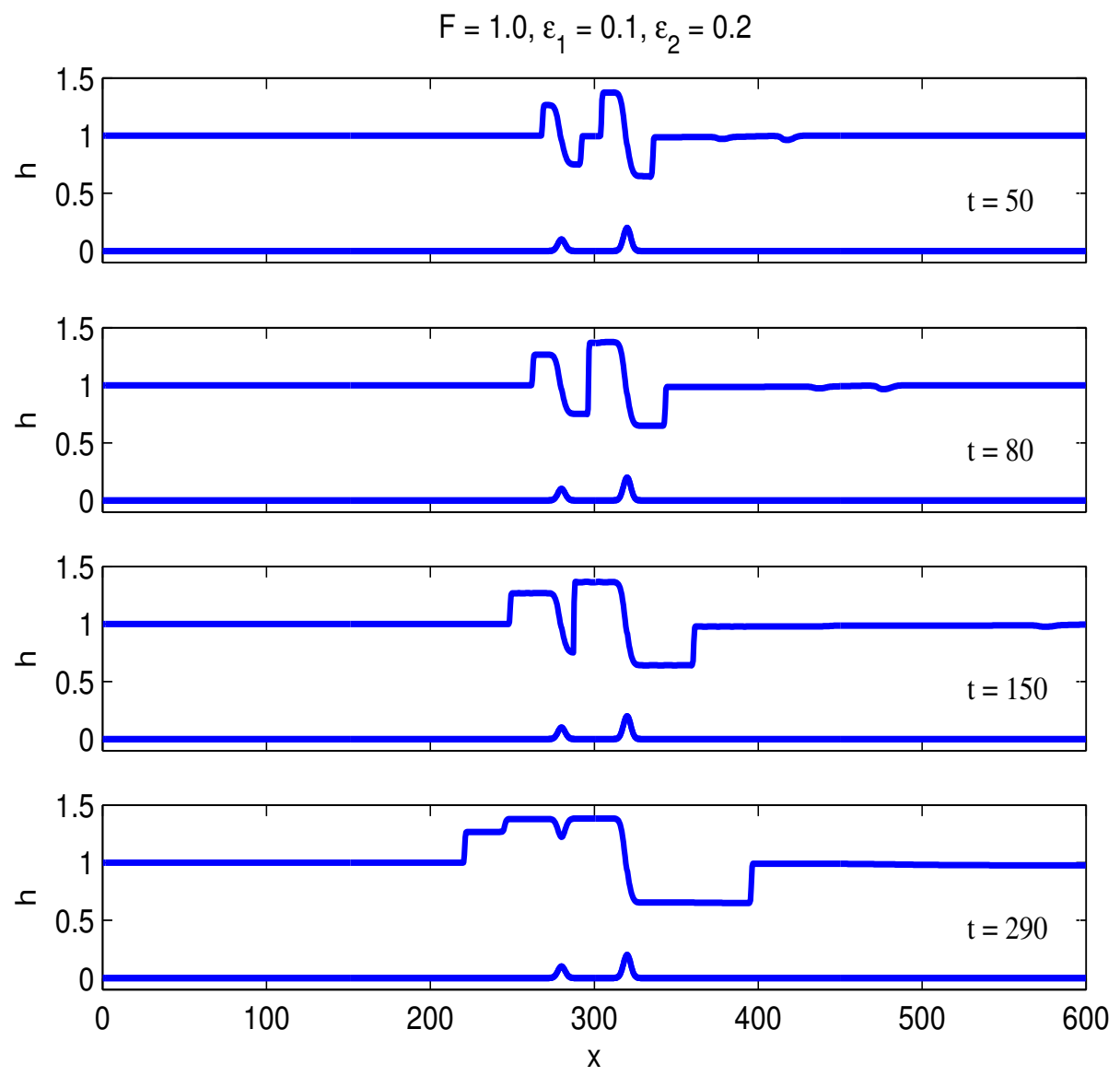

Figure 14: Simulations for $F=1.0, \epsilon_{1}=0.1, \epsilon_{2}=0.2$. 

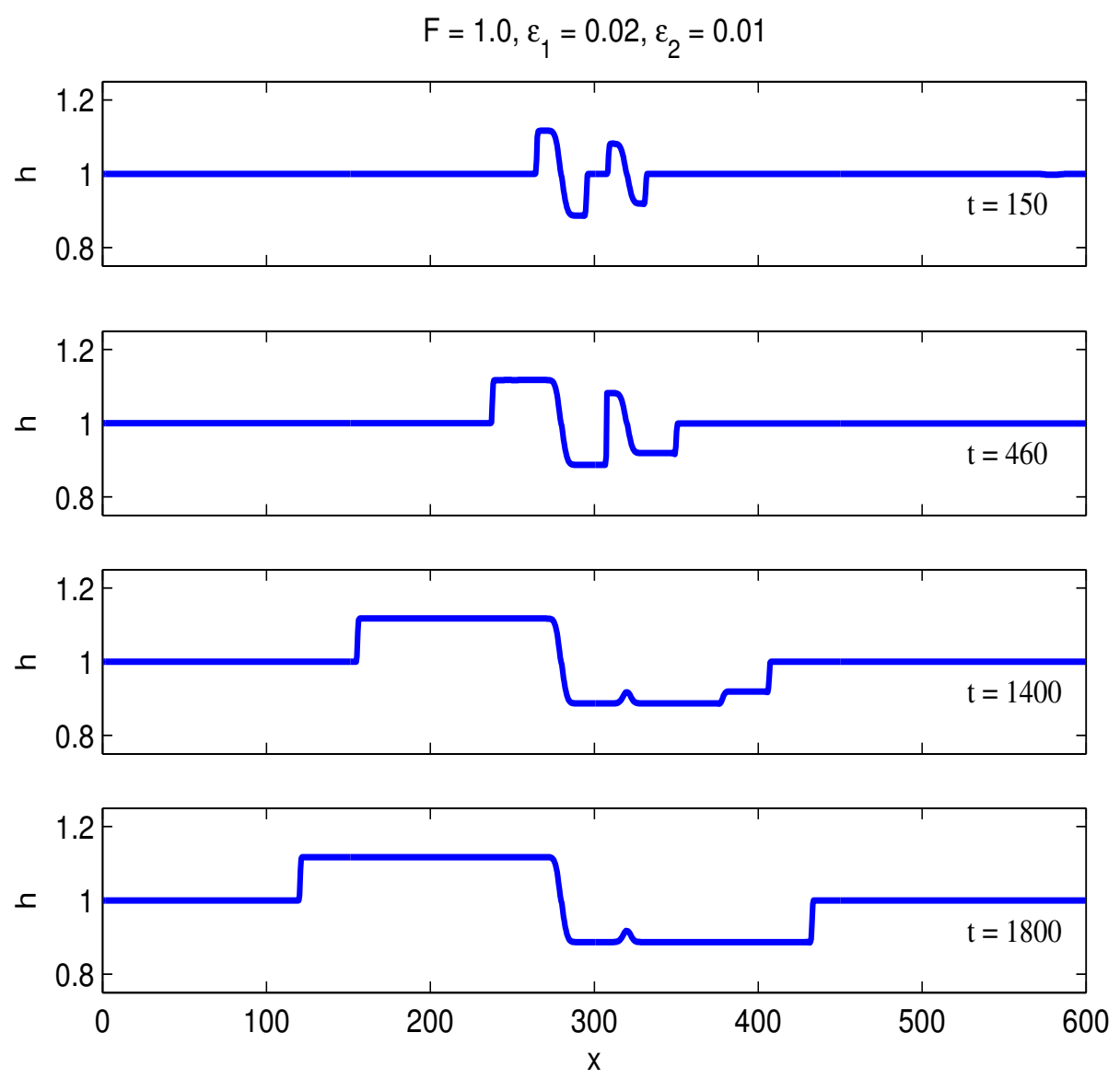

Figure 15: Simulations for $F=1.0, \epsilon_{1}=0.02, \epsilon_{2}=0.01$. 

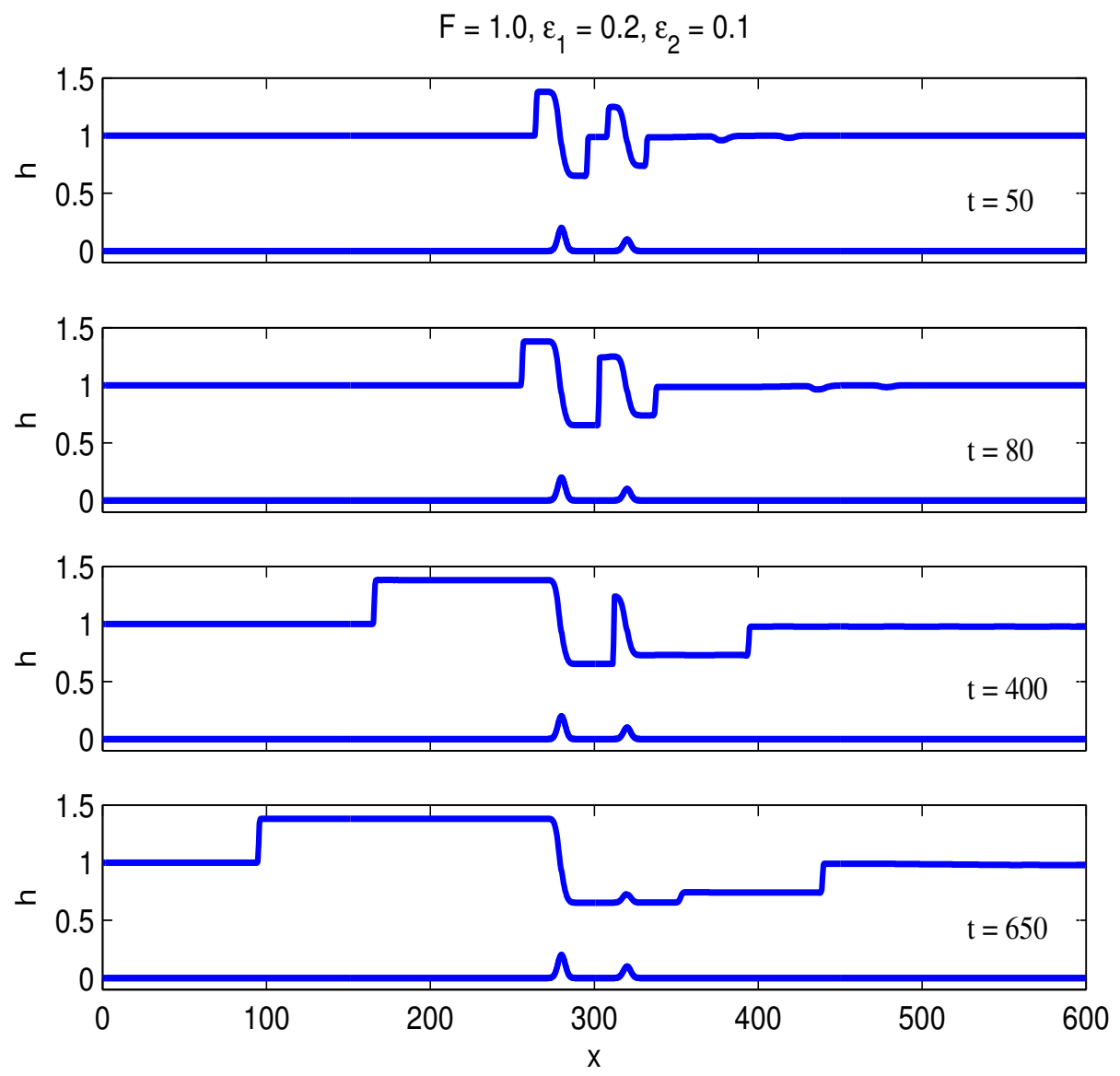

Figure 16: Simulations for $F=1.0, \epsilon_{1}=0.2$, and $\epsilon_{2}=0.1$. 